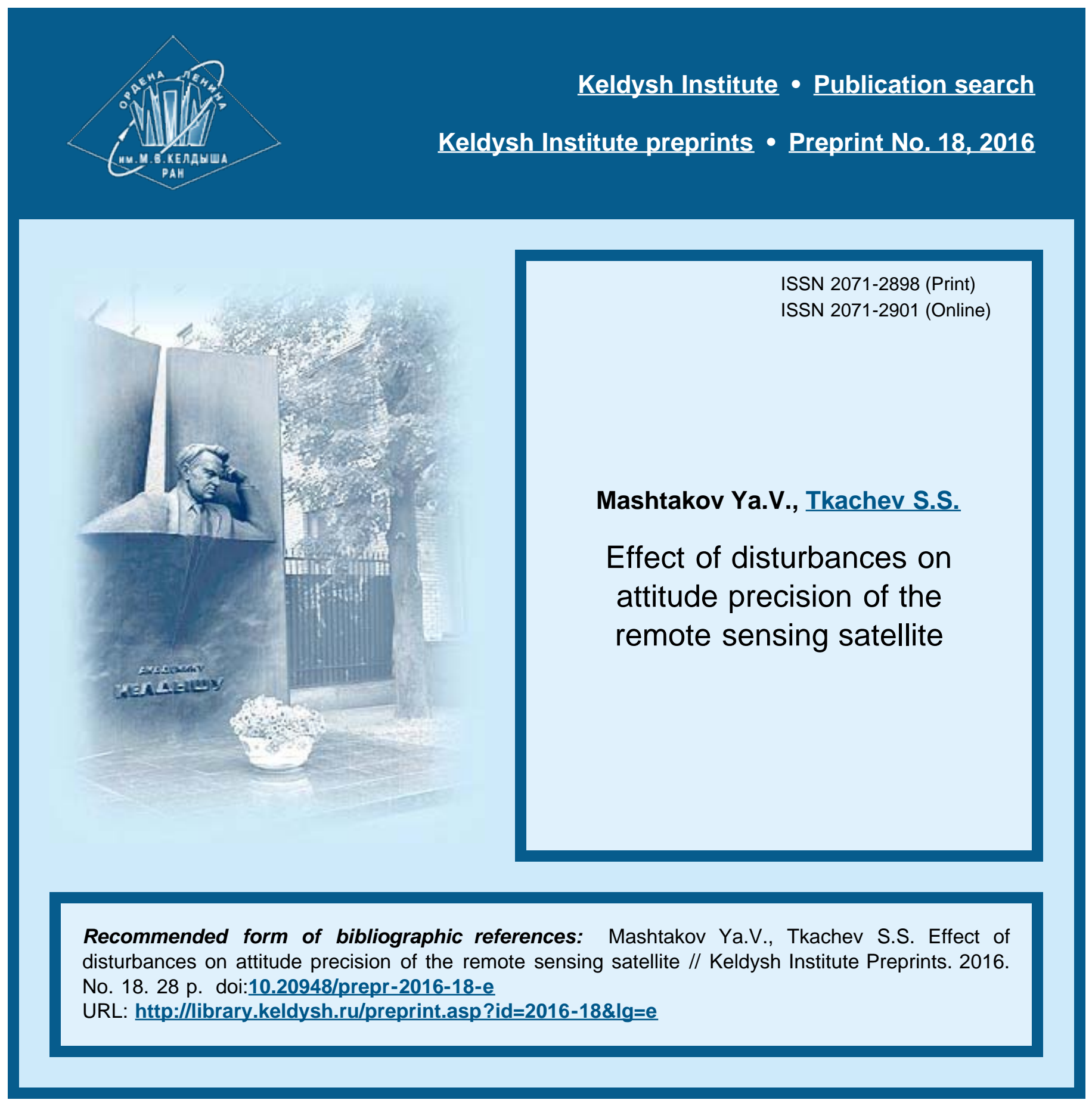




\author{
О рд н н а Л н и на \\ ИНСТИТУТ ПРИКЛАДНОЙ МАТЕМАТИКИ \\ имени М.В. Келдыша \\ Р ос ий ск ой а кадеми и наук
}

Y.V. Mashtakov, S.S. Tkachev

\title{
Effect of disturbances on attitude precision of the remote sensing satellite
}




\section{Маштаков Я.В., Ткачев С.С.}

Влияние возмущений на точность стабилизации спутника ДЗ3

Предложен алгоритм синтеза углового движения спутника дистанционного зондирования Земли (ДЗ3) для отслеживания маршрутов на поверхности Земли. Рассмотрено влияние неучтенных внешних возмущений на точность ориентации и стабилизации космического аппарата. Исследована связь между отклонением точки визирования и ошибки скорости бега изображения, точностью ориентации и стабилизации аппарата, а также кривизной снимаемой траектории и возможностями системы управления

Ключевые слова: дистанционное зондирование Земли, синтез углового движения, ПД-регулятор, точность ориентации

\section{Yaroslav Mashtakov, Stepan Tkachev}

Effect of disturbances on attitude precision of the remote sensing satellite

The angular motion synthesis algorithm for remote sensing satellite is developed. Effect of external disturbances on attitude accuracy is considered. Relations between attitude accuracy and image quality and trajectory curvature and control capabilities are investigated.

Key words: remote sensing, angular motion synthesis, PD-controller, attitude accuracy

The work was supported by RFBR grants 16-01-00739, 15-31-20058 


\section{Introduction}

Remote sensing satellites could be irreplaceable when hard to access areas are studied Usually, huge spacecrafts (SCs), such as «Resurs-P» [1] or «WorldView» [2] are used for remote sensing. They provide high-resolution pictures of Earth, but for near real-time monitoring (e.g. ice situation or forest fires monitoring) these satellites could be inappropriate due to long time intervals between two successive flybys over the region of interest. For this purpose, small satellites (less than hundred kilograms) on low Earth orbit (less than $400 \mathrm{~km}$ ) can be used. Development cost and time usually much lower for these satellites. Their capabilities of course are also lower, but it is possible to launch simultaneously several satellites that give qualitatively new options for remote sensing problems solutions.

In case of small remote sensing satellite, it is reasonable to fix camera with respect to the satellite body otherwise the complexity and development cost could be unacceptably high. Because of the limited size, it is impossible to install large highresolution and wide-angle cameras on such small satellites, so to increase the resolution of obtained images it is necessary to use small narrow-angle cameras that can cover much less territory but can provide images with satisfactory resolution. Therefore, ability to survey not only simple straight lines on the Earth surface but some complex routes might be quite useful.

It should be noticed that similar problem was discussed in papers of S.A. Butyrin and S.E.Somov [4-6], but they used numerical integration of kinematic equations for SC attitude quaternion. The main attention there is focused on velocities compensation of different image points which is very important in case of wide angle cameras. In case of small satellites with narrow angle cameras this problem is not crucial. In this paper problem of complex routes surveying is studied. This route is considered as three times continuously differentiable curve. Significant part of the paper is devoted to the study of disturbance influence on stabilization errors and image quality. Here, characteristics of image quality are image velocity w.r.t. camera sensing element (if its value is high, obtained image might be blurred) and observation point displacement (distance between the desirable point satellite "looking" at and the real one).

\section{Problem statement and coordinate systems}

\subsection{Problem statement}

In this paper, the problem of tracking the specified route on the Earth surface is considered. Following parameters are assumed to be known:

1) Orbital motion of the satellite, i.e. it's velocity and position at each moment

2) SC's tensor of inertia;

3) Parameters of the camera installed on the satellite: focal length and required image velocity; 
4) Parameters of the attitude control system: maximum and minimum control torque produced by reaction wheels and their maximum angular momentum;

5) Route on the Earth surface that is three times continuously differentiable parameterized curve.

Based on these data it is necessary to construct the angular motion of the satellite and suggest an algorithm of attitude control that can provide such motion. Moreover, it is required to provide the necessary accuracy of attitude stabilization even in the presence of unknown external disturbances (gravitational, magnetic and aerodynamical torques).

\subsection{Optical sensor features}

In this work, the CCD line is considered as optical sensor. It imposes some constraints on velocity of every image point in the camera focal plane (Fig. 1): it must be equal to a certain value and aligned with the normal to CCD line.

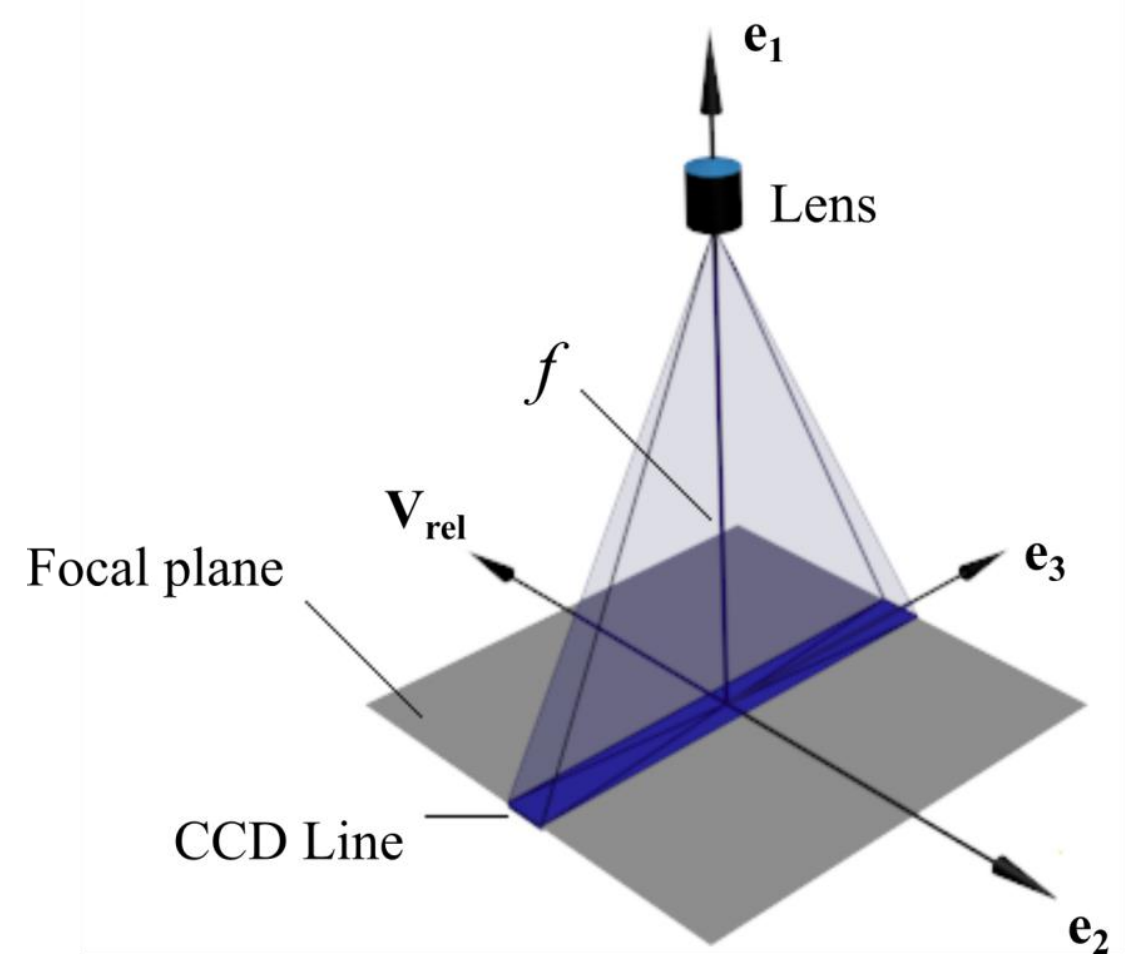

Fig. 1. Camera scheme

It is impossible to make all image points move with the same velocity in the focal plane due to already applied (see section 1.1) constraints for the angular velocity. Difference between velocities of central image point and the border one can be estimated as

$$
\Delta V \leq\left\|\left(\mathbf{\Omega}_{\mathrm{E}}-\boldsymbol{\omega}\right) \times \mathbf{r}\right\| \frac{f}{\rho} .
$$


Here $\mathbf{r}$ is vector from observation point to the swath border, $\boldsymbol{\Omega}_{\mathbf{E}}, \boldsymbol{\omega}$ are Earth and satellite angular velocity respectively, $f$ is camera focal length, $\rho$ is distance between the camera and observation point. If the swath width is considered to be 20 $\mathrm{km}, \rho=600 \mathrm{~km}, f=6 \mathrm{~m},|\omega|=10^{-3} \mathrm{rad} / \mathrm{s}$, difference in image velocities will be only $10^{-4} \mathrm{~m} / \mathrm{s}$. For example, required image velocity for the camera installed on the TabletSat-Aurora satellite (produced by SPUTNIX) is $5 \cdot 10^{-2} \mathrm{~m} / \mathrm{s}$. Thus, it is possible to apply constraint on image point velocity only to the central image point.

\subsection{Coordinate systems}

The following right-handed Cartesian coordinate systems are used (Fig. 2):

$O_{a} Y_{1} Y_{2} Y_{3}$ - Inertial Frame (IF): $O_{a}$ is located in the Earth center, $O_{a} Y_{1}$ axis is directed to the vernal equinox of $\mathrm{J} 2000$ epoch, $O_{a} Y_{3}$ is normal to equatorial plane;

$O_{a} Z_{1} Z_{2} Z_{3}$ - Greenwich Frame (GF): $O_{a}$ is located in the Earth center, $O_{a} Z_{1}$ locates in the equator plane and directed to the Prime Meridian, $O_{a} Z_{3}$ directed to the North Pole;

$O x_{1} x_{2} x_{3}$ - Body Frame (BF): $O$ located in the satellite center of mass, axes are its principal axes of inertia;

$C y_{1} y_{2} y_{3}$ - Camera Frame (CF): $C$ is located in the CCD line center, $C y_{1}$ aligned with camera optical axis, $C y_{2}$ is antiparallel to required image velocity;

$C X_{1} X_{2} X_{3}$ - Reference Frame (RF): $C$ is located in the CCD line center, $C X_{1}$ direction follows the route and constraint for velocity of central image point is satisfied (motion equations for these axes will be obtained in the next section).

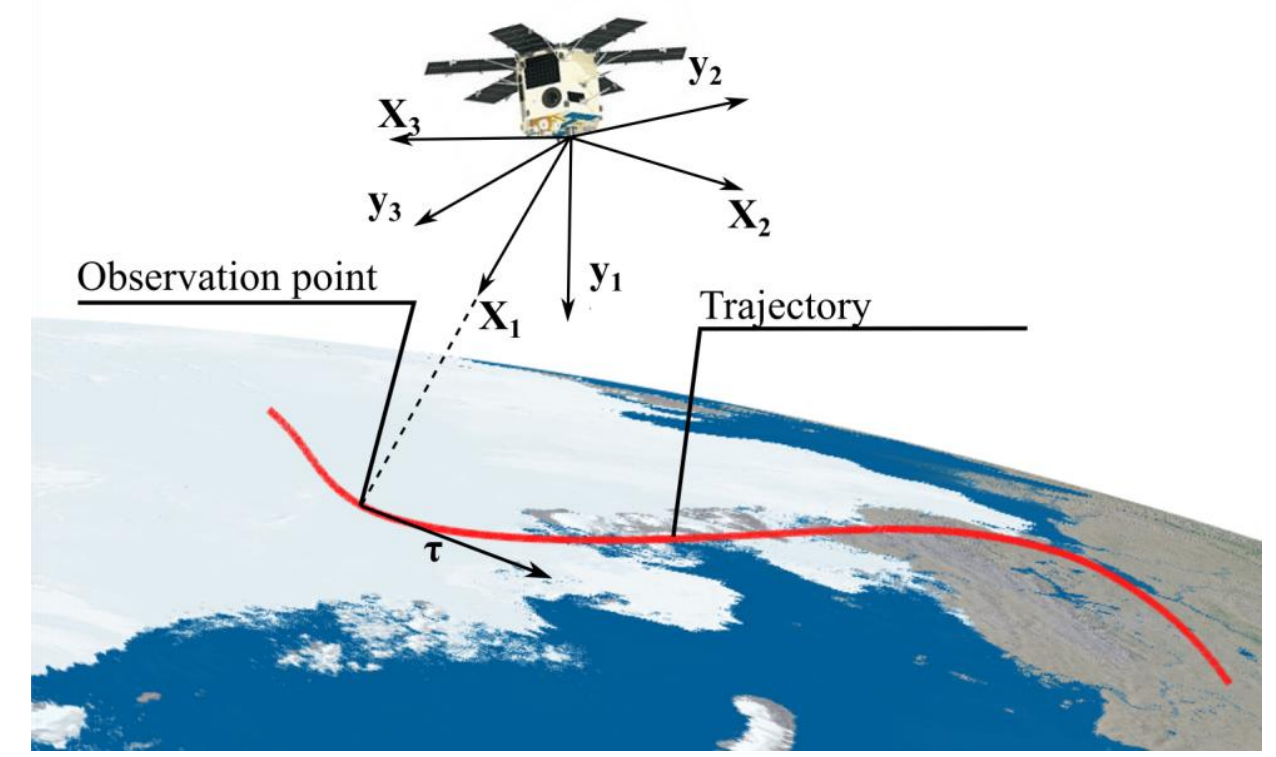

Fig. 2. Camera and Reference Frames 
Direction Cosine Matrices describe transition between these coordinate systems: $\mathbf{F}$ from RF to CF, $\mathbf{B}$ from IF to RF, $\mathbf{N}$ from GF to IF, $\mathbf{D}$ from IF to BF, $\mathbf{G}$ from CF to $\mathrm{BF}$ (the last matrix is constant, i.e. camera is rigidly fixed in the satellite).

\section{SC angular motion synthesis}

To construct reference angular motion (i.e. $\mathbf{B}(t)$ that provide surveying of the route and satisfy constraints imposed by CCD line camera) Poisson's equations for DCM are used

$$
\begin{gathered}
\dot{\mathbf{B}}=-[\boldsymbol{\omega}]_{\times} \mathbf{B}, \\
\mathbf{B}=\left(\begin{array}{c}
\mathbf{e}_{1}^{T} \\
\mathbf{e}_{2}^{T} \\
\mathbf{e}_{3}^{T}
\end{array}\right), \quad \dot{\mathbf{B}}=\left(\begin{array}{c}
\dot{\mathbf{e}}_{1}^{T} \\
\dot{\mathbf{e}}_{2}^{T} \\
\dot{\mathbf{e}}_{3}^{T}
\end{array}\right),
\end{gathered}
$$

where $\boldsymbol{\omega}$ is RF angular velocity, $\mathbf{e}_{i}$ are RF basis vectors. Further on dot denotes time derivative, and designation for skew-symmetric matrix of cross product is introduced: for any $\mathbf{y}=\left(\begin{array}{lll}y_{1} & y_{2} & y_{3}\end{array}\right)^{T}$

$$
[\mathbf{y}]_{\times}:=\left(\begin{array}{ccc}
0 & -y_{3} & y_{2} \\
y_{3} & 0 & -y_{1} \\
-y_{2} & y_{1} & 0
\end{array}\right) .
$$

So cross product of two vectors can be represented as matrix multiplication

$$
[\mathbf{a}]_{\times} \mathbf{b} \equiv \mathbf{a} \times \mathbf{b} .
$$

It was mentioned in Sec. 1.1 that route on the Earth surface can be described as three times continuously differentiable parameterized curve $\mathbf{r}_{p}(p)$, where $p$ is the curve parameter and $\mathbf{r}_{p}$ is the radius-vector of the point on this curve in GF. To construct angular motion of the satellite it is necessary to get this vector in IF:

$$
\tilde{\mathbf{r}}_{p}=\mathbf{N r} \mathbf{r}_{p},
$$

where $\mathbf{N}$, as was mentioned above, is transition matrix from GF to IF.

Image velocity constraints can be written as

$$
\begin{gathered}
\left(\mathbf{V}_{r e l}, \mathbf{e}_{3}\right) \frac{f}{\rho}=0, \\
\left(\mathbf{V}_{r e l}, \mathbf{e}_{2}\right) \frac{f}{\rho}=-V, \\
\mathbf{V}_{r e l}=\mathbf{V}_{p}-\mathbf{V}_{s}-\boldsymbol{\omega} \times\left(\mathbf{N} \mathbf{r}_{p}-\mathbf{r}_{s}\right)=\mathbf{\Omega}_{E} \times \mathbf{N} \mathbf{r}_{p}-\mathbf{V}_{s}-\boldsymbol{\omega} \times\left(\mathbf{N} \mathbf{r}_{p}-\mathbf{r}_{s}\right),
\end{gathered}
$$


where $\mathbf{V}_{p}, \mathbf{V}_{s}$ are observation point and $\mathrm{SC}$ velocities in IF respectively, $\boldsymbol{\omega}$ is $\mathrm{RF}$ angular velocity, $\mathbf{r}_{s}$ is radius-vector of SC center of mass, $\rho=\|\boldsymbol{\rho}\|=\left\|\mathbf{N} \mathbf{r}_{p}-\mathbf{r}_{s}\right\|$ is distance between the satellite and the observation point. From these equations it is possible to get some of angular velocity components

$$
\begin{gathered}
\omega_{2}=-\frac{\left(\boldsymbol{\Omega}_{E} \times \mathbf{N} \mathbf{r}_{p}-\mathbf{V}_{s}, \mathbf{e}_{3}\right)}{\rho}, \\
\omega_{3}=\frac{\left(\boldsymbol{\Omega}_{E} \times \mathbf{N} \mathbf{r}_{p}-\mathbf{V}_{s}, \mathbf{e}_{2}\right)}{\rho}+\frac{V}{f} .
\end{gathered}
$$

Equation for $\omega_{1}$ can be obtained from (1). RF basis vectors are defined as follows (the case of ideal control is considered, so CF and RF are aligned):

$$
\begin{gathered}
\mathbf{e}_{1}=\frac{\boldsymbol{\rho}}{\rho}, \\
\mathbf{e}_{3}=\frac{\mathbf{e}_{1} \times \mathbf{N} \boldsymbol{\tau}}{\left|\mathbf{e}_{1} \times \mathbf{N} \boldsymbol{\tau}\right|}, \boldsymbol{\tau}=\frac{\partial \mathbf{r}_{p}}{\partial p}, \\
\mathbf{e}_{2}=\mathbf{e}_{3} \times \mathbf{e}_{1} .
\end{gathered}
$$

Satellite size is much smaller than the distance between it and the observation point, so the vector between observation point and center of the optical sensor is considered equal to the one between observation point and satellite center of mass. Derivatives of the basis vectors can be described as follows:

$$
\begin{gathered}
\dot{\mathbf{e}}_{1}=\frac{\dot{\mathbf{N}} \mathbf{r}_{p}+\mathbf{N} \boldsymbol{\tau} \dot{p}-\mathbf{V}_{s}-\left(\dot{\mathbf{N}} \mathbf{r}_{p}+\mathbf{N} \boldsymbol{\tau} \dot{p}-\mathbf{V}_{s}, \mathbf{e}_{1}\right) \mathbf{e}_{1}}{\rho}, \\
\dot{\mathbf{e}}_{3}=\frac{\dot{\mathbf{e}}_{1} \times \mathbf{N} \boldsymbol{\tau}+\mathbf{e}_{1} \times(\dot{\mathbf{N}} \boldsymbol{\tau}+\dot{p} \mathbf{N})-\mathbf{e}_{3}\left(\dot{\mathbf{e}}_{1} \times \mathbf{N} \boldsymbol{\tau}+\mathbf{e}_{1} \times(\dot{\mathbf{N}} \boldsymbol{\tau}+\dot{p} \mathbf{N} \partial \boldsymbol{\tau} / \partial p), \mathbf{e}_{3}\right)}{\left|\mathbf{e}_{1} \times \mathbf{N} \boldsymbol{\tau}\right|}, \\
\dot{\mathbf{e}}_{2}=\dot{\mathbf{e}}_{3} \times \mathbf{e}_{1}+\mathbf{e}_{3} \times \dot{\mathbf{e}}_{1} .
\end{gathered}
$$

After substitution these equations in (1), one can get expressions for the angular velocity components:

$$
\begin{gathered}
\omega_{1}=\left(\dot{\mathbf{e}}_{2}, \mathbf{e}_{3}\right), \\
\omega_{2}=-\left(\dot{\mathbf{e}}_{1}, \mathbf{e}_{3}\right)=-\frac{\left(\dot{\mathbf{N}} \mathbf{r}_{p}+\mathbf{N} \boldsymbol{\tau} \dot{p}-\mathbf{V}_{s}, \mathbf{e}_{3}\right)}{\rho}, \\
\omega_{3}=\left(\dot{\mathbf{e}}_{1}, \mathbf{e}_{2}\right)=\frac{\left(\dot{\mathbf{N}} \mathbf{r}_{p}+\mathbf{N} \boldsymbol{\tau} \dot{p}-\mathbf{V}_{s}, \mathbf{e}_{2}\right)}{\rho}
\end{gathered}
$$


Comparing them with (3) and keeping in mind that $\dot{\mathbf{N}}=\left[\boldsymbol{\Omega}_{E}\right]_{\times} \mathbf{N}$ the following expressions can be obtained:

$$
\begin{gathered}
\frac{\left(\mathbf{N} \tau \dot{p}, \mathbf{e}_{3}\right)}{\rho}=\frac{\left(\mathbf{N} \boldsymbol{\tau} \dot{p}, \mathbf{e}_{1} \times \mathbf{N} \boldsymbol{\tau}\right)}{\rho\left|\mathbf{e}_{1} \times \mathbf{N} \boldsymbol{\tau}\right|} \equiv 0, \\
\dot{p} \frac{\left(\mathbf{N} \boldsymbol{\tau}, \mathbf{e}_{2}\right)}{\rho}=\frac{V}{f} .
\end{gathered}
$$

From the second expression differential equation for trajectory parameter $p$ is obtained

$$
\dot{p}=\frac{\rho(t, p) V}{f} \frac{1}{\left(\mathbf{N} \boldsymbol{\tau}(t, p), \mathbf{e}_{2}(t, p)\right)} .
$$

Solution of this equation and expressions (4) and (5) are completely define angular motion (i.e. angular velocity and attitude) of RF.

\section{Control synthesis, disturbances effect}

\subsection{PD-controller}

To implement obtained angular motion control algorithm based on Lyapunov function, also known as propotional derivative controller (PD-controller), is used [79]. The purpose of control is to match RF and CF.

Dynamical and kinematic equations of satellite angular motion are

$$
\begin{gathered}
\dot{\mathbf{K}}+\boldsymbol{\omega}_{a b s} \times \mathbf{K}=\mathbf{M}_{e x t}+\mathbf{M}_{c t r l}, \\
\dot{\mathbf{D}}=-\left[\boldsymbol{\omega}_{a b s}\right]_{\times} \mathbf{D}, \\
\dot{\mathbf{F}}=-\left[\mathbf{G}^{T} \boldsymbol{\omega}_{r e l}\right]_{\times} \mathbf{F},
\end{gathered}
$$

where $\mathbf{K}=\mathbf{J} \boldsymbol{\omega}_{a b s}$ is angular momentum of SC, $\boldsymbol{\omega}_{a b s}$ is its angular velocity (in BF), D is DCM from IF to BF, $\boldsymbol{\omega}_{r e l}=\boldsymbol{\omega}_{a b s}-\mathbf{G F G}^{T} \boldsymbol{\omega}_{r e f}, \boldsymbol{\omega}_{r e f}=\mathbf{G} \boldsymbol{\Omega}_{r e f}, \boldsymbol{\Omega}_{r e f}$ is reference angular velocity (in RF), $\mathbf{M}_{e x t}, \mathbf{M}_{c t r l}$ are external and control torques respectively. Due to $\mathbf{G}$ is being constant and that for every DCM $\mathbf{L}$ and vector $\mathbf{y}$ there is an equation

$$
[\mathbf{L y}]_{\times} \equiv \mathbf{L}[\mathbf{y}]_{\times} \mathbf{L}^{T}
$$

the last expression from (6) can be rewritten as

$$
\dot{\mathbf{A}}=-\left[\boldsymbol{\omega}_{r e l}\right]_{\times} \mathbf{A}, \mathbf{A}=\mathbf{G F G}^{T} .
$$

Lyapunov-candidate function is chosen in the form 


$$
V=\frac{1}{2}\left(\boldsymbol{\omega}_{\text {oтн }}, \mathbf{J} \boldsymbol{\omega}_{\text {oтн }}\right)+k_{a}(3-\operatorname{tr} \mathbf{A}), k_{a}>0 .
$$

This function is non-negative and equal to zero only when RF and $\mathrm{CF}$ are coincide. Considering (6), time derivative of Lyapunov-candidate function $V$ can be written as follows:

$$
\dot{V}=\frac{1}{2}\left(\boldsymbol{\omega}_{r e l}, \mathbf{J} \dot{\boldsymbol{\omega}}_{r e l}\right)-k_{a} \operatorname{tr} \dot{\mathbf{A}}=\left(\boldsymbol{\omega}_{r e l}, \mathbf{J} \dot{\boldsymbol{\omega}}_{a b s}+\mathbf{J}\left[\boldsymbol{\omega}_{r e l}\right]_{\times} \mathbf{A} \boldsymbol{\omega}_{r e f}-\mathbf{J A} \dot{\boldsymbol{\omega}}_{r e f}+k_{a} \mathbf{S}\right),
$$

where $\mathbf{S}=\left(\begin{array}{lll}a_{23}-a_{32} & a_{31}-a_{13} & a_{12}-a_{21}\end{array}\right), a_{i j}-$ elements of DCM A . If the following expression is fulfilled

$$
\mathbf{J} \dot{\boldsymbol{\omega}}_{a b s}+\mathbf{J}\left[\boldsymbol{\omega}_{r e l}\right]_{\times} \mathbf{A} \boldsymbol{\omega}_{r e f}-\mathbf{J A} \dot{\boldsymbol{\omega}}_{r e f}+k_{a} \mathbf{S}=-k_{\omega} \boldsymbol{\omega}_{r e l}, k_{\omega}>0,
$$

then $\dot{V} \leq 0$. Control torque in this case is

$$
\mathbf{M}_{c t r l}=-\mathbf{M}_{e x t}+\boldsymbol{\omega}_{a b s} \times \mathbf{J} \boldsymbol{\omega}_{a b s}-\mathbf{J}\left[\boldsymbol{\omega}_{r e l}\right]_{\times} \mathbf{A} \boldsymbol{\omega}_{r e f}+\mathbf{J A} \dot{\boldsymbol{\omega}}_{r e f}-k_{a} \mathbf{S}-k_{\omega} \boldsymbol{\omega}_{r e l} .
$$

Equations of relative angular motion of SC can be described as follows:

$$
\begin{gathered}
\mathbf{J} \dot{\boldsymbol{\omega}}_{r e l}+k_{\omega} \boldsymbol{\omega}_{r e l}+k_{a} \mathbf{S}=0, \\
\dot{\mathbf{A}}=-\left[\boldsymbol{\omega}_{r e l}\right]_{\times} \mathbf{A} .
\end{gathered}
$$

It is easy to see that there is no whole trajectories that belong to the set $\left(\mathbf{A}, \boldsymbol{\omega}_{\text {rel }}\right): \dot{V}=0$ except $\mathbf{A} \equiv \mathbf{E}_{3 \times 3}, \boldsymbol{\omega}_{\text {rel }} \equiv 0$. Hence, according to Barbashin-Krasovsky theorem[10], $\mathbf{A} \equiv \mathbf{E}_{3 \times 3}, \boldsymbol{\omega}_{\text {rel }} \equiv 0$ is asymptotically stable.

\subsection{Disturbances effect}

There is $\mathbf{M}_{\text {ext }}$ in expression for control torque (7) that is the sum of all external torques. Unfortunately, the model of external torques usually not sufficiently precise or too difficult to calculate it on-board, so the system (8) must be rewritten as follows:

$$
\begin{gathered}
\mathbf{J} \dot{\boldsymbol{\omega}}_{r e l}+k_{\omega} \boldsymbol{\omega}_{r e l}+k_{a} \mathbf{S}=\mathbf{M}_{d i s t}, \\
\dot{\mathbf{A}}=-\left[\boldsymbol{\omega}_{r e l}\right]_{\times} \mathbf{A},
\end{gathered}
$$

where $\mathbf{M}_{\text {dist }}$ represents sum of all the torques that do not taken into account during control synthesis. In this work aerodynamical and gravitational torques, as well as SC tensor of inertia inaccuracy, are considered as disturbances. 


$$
\begin{gathered}
\mathbf{M}_{a t m}=-\frac{\rho_{a t m} C_{\mathrm{x}} S v^{2} r_{a} \mathbf{e}_{a} \times \mathbf{e}_{v}}{2},\left|\mathbf{e}_{a}\right|=\left|\mathbf{e}_{v}\right|=1, \\
\mathbf{M}_{g r a v}=3 \frac{\mu_{E}}{r_{s}^{5}} \mathbf{r}_{s} \times \mathbf{J} \mathbf{r}_{s}, \\
\mathbf{M}_{i n}=\boldsymbol{\omega}_{a b s} \times \delta \mathbf{J} \boldsymbol{\omega}_{a b s}+\delta \mathbf{J}\left(-\left[\boldsymbol{\omega}_{r e l}\right]_{\times} \mathbf{A} \boldsymbol{\omega}_{r e f}+\mathbf{A} \dot{\boldsymbol{\omega}}_{r e f}\right),
\end{gathered}
$$

where $\rho_{a t m}$ is atmosphere density, $C_{\mathrm{x}}$ is drag coefficient, $S$ is SC cross-sectional area, $v \mathbf{e}_{v}$ is SC velocity, $r_{a} \mathbf{e}_{a}$ is radius-vector between SC center of mass and center of pressure, $\mu_{E}$ is Earth gravitational parameter, $\delta \mathbf{J}$ is tensor of inertia inaccuracy. It is necessary to mention that suggested technique is universal and can be applied to any other disturbance.

To further analysis, it is convenient to rewrite equations of relative motion in dimensionless form:

$$
\begin{gathered}
\mathbf{I} \mathbf{\Omega}^{\prime}+K_{a} \mathbf{S}+K_{\omega} \mathbf{\Omega}=\varepsilon_{1} \mathbf{M}_{1}+\varepsilon_{2} \mathbf{M}_{2}+\varepsilon_{3} \mathbf{M}_{3}, \\
\mathbf{A}^{\prime}=-[\mathbf{\Omega}]_{\times} \mathbf{A}, \\
\mathbf{\Omega}=\boldsymbol{\omega}_{r e l} \sqrt{\frac{M_{0}}{J_{0}}}, q=t \sqrt{\frac{M_{0}}{J_{0}}}, \mathbf{I}=\frac{\mathbf{J}}{J_{0}}, \\
\delta \mathbf{I}=\frac{\delta \mathbf{J}}{J_{0}}, K_{a}=\frac{k_{a}}{M_{0}}, K_{\omega}=\frac{k_{\omega}}{\sqrt{J_{0} M_{0}}}, \\
\varepsilon_{1}=-\frac{\rho_{a t m} C_{\mathrm{x}} S v^{2} r_{a}}{2 M_{0}}, \mathbf{M}_{1}=\mathbf{e}_{a} \times \mathbf{e}_{v}, \\
\varepsilon_{2}=3 \frac{\mu_{E} J_{0}}{r_{s}^{3} M_{0}}, \mathbf{M}_{2}=\frac{\mathbf{r}_{s}}{r_{s}} \times \mathbf{I} \frac{\mathbf{r}_{s}}{r_{s}}, \\
\varepsilon_{3}=\frac{J_{0} \omega_{a b s}^{2}}{M_{0}}, \mathbf{M}_{3}=\frac{\boldsymbol{\omega}_{a b s} \times \delta \mathbf{I} \boldsymbol{\omega}_{a b s}}{\omega_{a b s}^{2}}+\frac{\delta \mathbf{I}}{\omega_{a b s}^{2}}\left(-\left[\boldsymbol{\omega}_{r e l}\right]_{\times} \mathbf{A} \boldsymbol{\omega}_{r e f}+\mathbf{A} \dot{\boldsymbol{\omega}}_{r e f}\right),
\end{gathered}
$$

where dash denotes derivative with respect to $q, M_{0}$ is maximum control torque that can be implemented by attitude control system, $J_{0}=\max _{i, j}\left(J_{i j}\right), \varepsilon_{i}$ is characteristic value of i-th disturbance torque.

Here the case of $\varepsilon_{i}<<1$ is considered, so the difference between $\mathrm{CF}$ and RF is small. Hence, it is possible to linearize $\mathrm{SC}$ relative motion equations in neighborhood of undisturbed stability point $\alpha_{i}=0, \boldsymbol{\omega}_{\text {rel }}=0$. DCM A can be described by Euler angles, which are the sequence of rotation angles $\alpha_{2}, \alpha_{3}, \alpha_{1}$ around the second, the third and the first axis respectively: 


$$
\begin{array}{ll}
a_{11}=\cos \alpha_{2} \cos \alpha_{3}, & a_{12}=\sin \alpha_{3}, \\
a_{21}=-\cos \alpha_{2} \sin \alpha_{3} \cos \alpha_{1}+\sin \alpha_{2} \sin \alpha_{1}, & a_{22}=\cos \alpha_{3} \cos \alpha_{1}, \\
a_{31}=\cos \alpha_{2} \sin \alpha_{3} \sin \alpha_{1}+\sin \alpha_{2} \cos \alpha_{1}, & a_{23}=\sin \alpha_{2} \sin \alpha_{3} \cos \alpha_{1}+\cos \alpha_{2} \sin \alpha_{1}, \\
a_{31}=\cos \alpha_{2} \sin \alpha_{3} \sin \alpha_{1}+\sin \alpha_{2} \cos \alpha_{1}, \\
a_{32}=-\cos \alpha_{3} \sin \alpha_{1}, \\
a_{33}=-\sin \alpha_{2} \sin \alpha_{3} \sin \alpha_{1}+\cos \alpha_{2} \cos \alpha_{1} .
\end{array}
$$

Linearization in the neighborhood of $\alpha_{1}=\alpha_{2}=\alpha_{3}=0$ gives expressions for $\mathbf{A}$ and $\mathbf{S}$

$$
\begin{aligned}
& \mathbf{A}=\left(\begin{array}{ccc}
1 & \alpha_{3} & -\alpha_{2} \\
-\alpha_{3} & 1 & \alpha_{1} \\
\alpha_{2} & -\alpha_{1} & 1
\end{array}\right), \\
& \mathbf{S}=2\left(\begin{array}{lll}
\alpha_{1} & \alpha_{2} & \alpha_{3}
\end{array}\right)^{T}=2 \boldsymbol{\alpha} .
\end{aligned}
$$

Angular velocity is described as follows:

$$
\mathbf{\Omega}=\boldsymbol{\alpha}^{\prime}, \mathbf{\Omega}^{\prime}=\boldsymbol{\alpha}^{\prime \prime} .
$$

Hence, linearized system of equations is

$$
\mathbf{I} \boldsymbol{\alpha}^{\prime \prime}+K_{\omega} \boldsymbol{\alpha}^{\prime}+2 K_{a} \boldsymbol{\alpha}=\varepsilon_{1} \mathbf{M}_{1}+\varepsilon_{2} \mathbf{M}_{2}+\varepsilon_{3} \mathbf{M}_{3} .
$$

Since solution of this system can be described as a sum of partial solutions, it is suitable to consider the general system

$$
\mathbf{I} \boldsymbol{\alpha}^{\prime \prime}+K_{\omega} \boldsymbol{\alpha}^{\prime}+2 K_{a} \boldsymbol{\alpha}=\varepsilon \mathbf{M},
$$

where $\varepsilon$ is small parameter. Since $\varepsilon<<1$, it is possible to use Poincare method [11] of expanding solution into a series in power of $\varepsilon$

$$
\boldsymbol{\alpha}=\boldsymbol{\alpha}^{0}+\varepsilon \boldsymbol{\alpha}^{1}+\varepsilon^{2} \boldsymbol{\alpha}^{2}+\ldots
$$

So, the system of relative motion equations is

$$
\begin{gathered}
\mathbf{I}\left(\boldsymbol{\alpha}^{0}\right)^{\prime \prime}+K_{\omega}\left(\boldsymbol{\alpha}^{0}\right)^{\prime}+2 K_{a} \boldsymbol{\alpha}^{0}=0, \\
\mathbf{I}\left(\boldsymbol{\alpha}^{1}\right)^{\prime \prime}+K_{\omega}\left(\boldsymbol{\alpha}^{1}\right)^{\prime}+2 K_{a} \boldsymbol{\alpha}^{1}=\mathbf{M}\left(q, \boldsymbol{\alpha}^{0}\right),
\end{gathered}
$$

Since $\mathbf{I}$ is diagonal, this system can be rewritten in the following form (equations for the second and greater order of $\varepsilon$ are omitted here) 


$$
\begin{gathered}
I_{i i}\left(\alpha_{i}^{0}\right)^{\prime \prime}+K_{\omega}\left(\alpha_{i}^{0}\right)^{\prime}+2 K_{a} \alpha_{i}^{0}=0, \\
I_{i i}\left(\alpha_{i}^{1}\right)^{\prime \prime}+K_{\omega}\left(\alpha_{i}^{1}\right)^{\prime}+2 K_{a} \alpha_{i}^{1}=M\left(q, \boldsymbol{\alpha}^{0}\right), i=1,2,3 .
\end{gathered}
$$

Using notation $2 \gamma_{i}=\frac{K_{\omega}}{I_{i i}}, \theta_{i}^{2}=\frac{2 K_{a}}{I_{i i}}, f_{i}=\frac{M\left(q, \boldsymbol{\alpha}_{0}\right)}{I_{i i}}$, equations of relative motion can be written as follows:

$$
\begin{gathered}
\left(\alpha_{i}^{0}\right)^{\prime \prime}+2 \gamma_{i}\left(\alpha_{i}^{0}\right)^{\prime}+\theta_{i}^{2} \alpha_{i}^{0}=0, \\
\left(\alpha_{i}^{1}\right)^{\prime \prime}+2 \gamma_{i}\left(\alpha_{i}^{1}\right)^{\prime}+\theta_{i}^{2} \alpha_{i}^{1}=f_{i}, i=1,2,3 .
\end{gathered}
$$

Solutions for these systems are depend on the relations between $\gamma_{i}$ and $\theta_{i}$.

1. Case $\gamma_{i}^{2}-\theta_{i}^{2}>0$

In this case solutions of the zero order system are

$$
\alpha_{i}^{0}=A \exp \left(\left(-\gamma_{i}+\varphi_{i}\right) q\right)+B \exp \left(\left(-\gamma_{i}-\varphi_{i}\right) q\right), \varphi_{i}=\sqrt{\gamma_{i}^{2}-\theta_{i}^{2}} .
$$

After substitution it in $f_{i}\left(q, \boldsymbol{\alpha}_{0}\right)$, one can obtain $f_{i}\left(q, \boldsymbol{\alpha}^{0}(q)\right) \rightarrow f_{i}(q)$. Parameters variation method is used to obtain solutions of the first order system. Differential equations for these parameters are

$$
\begin{gathered}
A^{\prime} \exp \left(\left(-\gamma_{i}+\varphi_{i}\right) q\right)+B^{\prime} \exp \left(\left(-\gamma_{i}-\varphi_{i}\right) q\right)=0, \\
\left(-\gamma_{i}+\varphi_{i}\right) A^{\prime} \exp \left(\left(-\gamma_{i}+\varphi_{i}\right) q\right)+ \\
+\left(-\gamma_{i}-\varphi_{i}\right) B^{\prime} \exp \left(\left(-\gamma_{i}-\varphi_{i}\right) q\right)=f_{i}(q) .
\end{gathered}
$$

Their solutions are

$$
\begin{gathered}
A^{\prime}=\frac{f_{i}(q) \exp \left(\left(-\gamma_{i}-\varphi_{i}\right) q\right)}{2 \varphi_{i}}, \\
B^{\prime}=-\frac{f_{i}(q) \exp \left(\left(-\gamma_{i}+\varphi_{i}\right) q\right)}{2 \varphi_{i}},
\end{gathered}
$$

and solutions of first order system are

$$
\alpha_{i}^{1}=\int_{0}^{q} \frac{f_{i}(p)}{\varphi_{i}} \exp \left(-\gamma_{i}(q-p)\right) \operatorname{sh}\left(\varphi_{i}(q-p)\right) d p
$$

2. Case $\gamma_{i}^{2}-\theta_{i}^{2}<0$

Solution of the zero order system is

$$
\alpha_{i}^{0}=A \exp \left(-\gamma_{i} q\right) \cos \left(\psi_{i} q\right)+\operatorname{Bexp}\left(-\gamma_{i} q\right) \sin \left(\psi_{i} q\right), \psi_{i}=\sqrt{\theta^{2}-\gamma^{2}} .
$$


System of differential equations for $A, B$ can be written as follows:

$$
\begin{gathered}
A^{\prime} \exp \left(-\gamma_{i} q\right) \cos \left(\psi_{i} q\right)+B^{\prime} \exp \left(-\gamma_{i} q\right) \sin \left(\psi_{i} q\right)=0, \\
-\psi_{i} A^{\prime} \exp \left(-\gamma_{i} q\right) \sin \left(\psi_{i} q\right)+\psi_{i} B^{\prime} \exp \left(-\gamma_{i} q\right) \cos \left(\psi_{i} q\right)=f_{i}(q) .
\end{gathered}
$$

Final expressions for $A^{\prime}, B^{\prime}$ are

$$
\begin{aligned}
A^{\prime} & =-\frac{f_{i}(q) \exp \left(\gamma_{i} q\right)}{\psi_{i}} \sin \left(\psi_{i} q\right), \\
B^{\prime} & =\frac{f_{i}(q) \exp \left(\gamma_{i} q\right)}{\psi_{i}} \cos \left(\psi_{i} q\right),
\end{aligned}
$$

and the solution of the first order system is

$$
\alpha_{i}^{1}=\frac{1}{\psi_{i}} \int_{0}^{q} f_{i}(p) \exp \left(\gamma_{i}(p-q)\right) \sin \left(\psi_{i}(q-p)\right) d p
$$

3. Case $\gamma_{i}^{2}-\theta_{i}^{2}=0$

Solution of zero order system is

$$
\alpha_{i}^{0}=A \exp \left(-\gamma_{i} q\right)+B q \exp \left(-\gamma_{i} q\right)
$$

System of equations for $A^{\prime}, B^{\prime}$ is

$$
\begin{gathered}
A^{\prime} \exp \left(-\gamma_{i} q\right)+B^{\prime} q \exp \left(-\gamma_{i} q\right)=0, \\
-\gamma_{i} A^{\prime} \exp \left(-\gamma_{i} q\right)+\left(1-\gamma_{i} q\right) B^{\prime} \exp \left(-\gamma_{i} q\right)=f_{i}(q),
\end{gathered}
$$

and its solution is

$$
\begin{aligned}
& B^{\prime}=f_{i}(q) \exp \left(\gamma_{i} q\right), \\
& A^{\prime}=-q f_{i}(q) \exp \left(\gamma_{i} q\right) .
\end{aligned}
$$

First order system solution in this case can be described as follows:

$$
\alpha_{i}^{1}=\int_{0}^{q} f_{i}(p) \exp \left(-\gamma_{i}(q-p)\right)(q-p) d p
$$

Usually it is impossible to integrate obtained expressions, but for remote sensing missions maximum angles deviations and stabilization errors are only important.

\subsection{Estimation of maximum deviations}

Expressions for $\alpha_{i}^{0}, \alpha_{i}^{1}$ are used to estimate maximum attitude deviation and stabilization error of the satellite. Since $\alpha_{i} \equiv 0$ is asymptotic stable in undisturbed motion, it is reasonable to consider $\alpha_{i}^{0} \equiv 0$.

1. Case $\gamma_{i}^{2}-\theta_{i}^{2}>0$ 
As was shown in previous section, in this case equations for Euler angles can be described as follows (with an accuracy up to $\varepsilon^{2}$ )

$$
\alpha_{i}(t)=\varepsilon \int_{0}^{q} \frac{f_{i}(p)}{\varphi_{i}} \exp \left(-\gamma_{i}(q-p)\right) \operatorname{sh}\left(\varphi_{i}(q-p)\right) d p .
$$

Angular velocity (in dimensionless form) is

$$
\begin{gathered}
\Omega_{i}=\left(\alpha_{i}\right)^{\prime}=-\varepsilon \int_{0}^{q} \frac{\gamma_{i}}{\varphi_{i}} f_{i}(p) \exp \left(-\gamma_{i}(q-p)\right) \operatorname{sh}\left(\varphi_{i}(q-p)\right) d p+ \\
+\varepsilon \int_{0}^{q} f_{i}(p) \exp \left(-\gamma_{i}(q-p)\right) \operatorname{ch}\left(\varphi_{i}(q-p)\right) d p .
\end{gathered}
$$

Hence, introducing notation $f_{\max }=\varepsilon \max _{t}\left(\left|f_{i}(q)\right|\right)$, estimations for attitude deviations and stabilization errors are

$$
\begin{gathered}
\left|\alpha_{i}(q)\right| \leq f_{\max }\left|\int_{0}^{q} \frac{1}{\varphi_{i}} \exp \left(-\gamma_{i}(q-p)\right) \operatorname{sh}\left(\varphi_{i}(q-p)\right) d p\right| \leq \\
\leq f_{\max }\left|\int_{0}^{\infty} \frac{1}{\varphi_{i}} \exp \left(-\gamma_{i} x\right) \operatorname{sh}\left(\varphi_{i} x\right) d x\right|=f_{\max } \frac{1}{\gamma_{i}^{2}-\varphi_{i}^{2}} \\
\left|\Omega_{i}\right| \leq \frac{f_{\max }}{\varphi_{i}} \int_{0}^{\infty}\left|-\gamma_{i} \exp \left(-\gamma_{i} x\right) \operatorname{sh}\left(\varphi_{i} x\right)+\varphi_{i} \exp \left(-\gamma_{i} x\right) \operatorname{ch}\left(\varphi_{i} x\right)\right| d x= \\
=2 f_{\max }\left(\frac{\gamma_{i}+\varphi_{i}}{\gamma_{i}-\varphi_{i}}\right)^{-\frac{\gamma_{i}}{2 \varphi_{i}}} \frac{1}{\sqrt{\gamma_{i}^{2}-\varphi_{i}^{2}}}
\end{gathered}
$$

These expressions can be rewritten in dimension form:

$$
\begin{gathered}
\left|\alpha_{i}(t)\right| \leq \frac{M_{\max }}{2 k_{a}}, \\
\left|\omega_{i}(t)\right| \leq \frac{2 M_{\max }}{\sqrt{2 J_{i i} k_{a}}}\left(\frac{k_{\omega}+\sqrt{k_{\omega}^{2}-8 k_{a} J_{i i}}}{k_{\omega}-\sqrt{k_{\omega}^{2}-8 k_{a} J_{i i}}}\right)^{\frac{-k_{\omega}}{2 \sqrt{k_{\omega}^{2}-8 k_{a} J_{i i}}}},
\end{gathered}
$$

where $M_{\max }=f_{\max } M_{0} I_{i i}$ is maximum value of the disturbance torque.

2. Case $\gamma_{i}^{2}-\theta_{i}^{2}<0$

Equations for Euler angles and relative angular velocity are 


$$
\begin{aligned}
\alpha_{i}(q)= & \frac{\varepsilon}{\psi_{i}} \int_{0}^{q} f_{i}(p) \exp \left(\gamma_{i}(p-q)\right) \sin \left(\psi_{i}(q-p)\right) d p, \\
\Omega_{i}(q)= & -\varepsilon \frac{\gamma_{i}}{\psi_{i}} \int_{0}^{q} f_{i}(p) \exp \left(\gamma_{i}(p-q)\right) \sin \left(\psi_{i}(q-p)\right) d p+ \\
& +\varepsilon \int_{0}^{q} f_{i}(p) \exp \left(\gamma_{i}(p-q)\right) \cos \left(\psi_{i}(q-p)\right) d p .
\end{aligned}
$$

To estimate these integrals the following expression is used:

$$
\begin{aligned}
& \int_{0}^{\infty} \exp (-a x)|\sin (x)| d x=\sum_{k=0}^{\infty}(-1)^{k} \int_{\pi k}^{\pi(k+1)} \exp (-a x) \sin (x) d x= \\
& =\int_{0}^{\pi} \exp (-a x) \sin (x) d x \sum_{k=0}^{\infty} \exp (-a \pi k)=\operatorname{cth}\left(\frac{a \pi}{2}\right) \frac{1}{a^{2}+1} .
\end{aligned}
$$

Hence, estimation for maximum attitude and stabilization errors are

$$
\begin{aligned}
& \left|\alpha_{i}(q)\right| \leq \frac{f_{\max }}{\psi_{i}} \int_{0}^{q} \exp \left(\gamma_{i}(p-q)\right)\left|\sin \left(\psi_{i}(q-p)\right)\right| d p \leq \\
& \leq \frac{f_{\max }}{\psi_{i}^{2}} \int_{0}^{\infty} \exp \left(-\frac{\gamma_{i}}{\psi_{i}} x\right)|\sin (x)| d p=\operatorname{cth}\left(\frac{\gamma_{i} \pi}{2 \psi_{i}}\right) \frac{f_{\max }}{\gamma_{i}^{2}+\psi_{i}^{2}}, \\
& \left|\Omega_{i}(q)\right|=\left|-\varepsilon \frac{\sqrt{\gamma_{i}^{2}+\psi_{i}^{2}}}{\psi_{i}} \int_{0}^{q} f(p) \exp \left(\gamma_{i}(p-q)\right) \sin \left(\psi_{i}(q-p)-\zeta\right)\right| \leq \\
& \leq f_{\max } \frac{\sqrt{\gamma_{i}^{2}+\psi_{i}^{2}}}{\psi_{i}^{2}} \exp \left(-\frac{\gamma_{i} \zeta}{\psi_{i}}\right)\left(\int_{-\zeta}^{\infty} \exp \left(-\frac{\gamma_{i} x}{\psi_{i}}\right)|\sin (x)| d x\right) \leq \\
& \leq \frac{f_{\max }}{\sqrt{\gamma_{i}^{2}+\psi_{i}^{2}}} \exp \left(-\frac{\gamma_{i} \zeta}{\psi_{i}}\right)\left(1+\operatorname{cth}\left(\frac{\gamma_{i} \pi}{2 \psi_{i}}\right)\right),
\end{aligned}
$$

where

$$
\zeta=\arccos \left(\frac{\gamma_{i}}{\sqrt{\gamma_{i}^{2}+\psi_{i}^{2}}}\right), 0 \leq \zeta \leq \frac{\pi}{2} .
$$

They can be rewritten in dimension form as follows: 


$$
\begin{gathered}
\left|\alpha_{i}(t)\right| \leq M_{\max } \operatorname{cth}\left(\frac{\pi k_{\omega}}{2 \sqrt{8 k_{a} J_{i i}-k_{\omega}^{2}}}\right) \frac{1}{2 k_{a}}, \\
\left|\omega_{i}(t)\right| \leq \frac{M_{\max }}{\sqrt{2 J_{i i} k_{a}}} \exp \left(\frac{-k_{\omega}}{\sqrt{8 k_{a} J_{i i}-k_{\omega}^{2}}} \arccos \left(\frac{k_{\omega}}{\sqrt{8 k_{a} J_{i i}}}\right)\right)\left(1+\operatorname{cth}\left(\frac{\pi k_{\omega}}{2 \sqrt{8 k_{a} J_{i i}-k_{\omega}^{2}}}\right)\right) .
\end{gathered}
$$

3. Case $\gamma_{i}^{2}-\theta_{i}^{2}=0$

Equations for Euler angles and relative angular velocity are

$$
\begin{aligned}
& \alpha_{i}=\varepsilon \int_{0}^{q} f_{i}(p) \exp \left(-\gamma_{i}(q-p)\right)(q-p) d p \\
& \Omega_{i}=\varepsilon \int_{0}^{q} f_{i}(p) \exp \left(-\gamma_{i}(q-p)\right)\left(1-\gamma_{i}(q-p)\right) d p .
\end{aligned}
$$

They can be estimated as follows:

$$
\begin{aligned}
& \left|\alpha_{i}\right| \leq f_{\max } \int_{0}^{\infty} \exp \left(-\gamma_{i} x\right) x d x=\frac{f_{\max }}{\gamma_{i}^{2}}, \\
& \left|\Omega_{i}\right| \leq f_{\max } \int_{0}^{\infty} \exp \left(-\gamma_{i} x\right)\left(1-\gamma_{i} x\right) \mid d x=2 \frac{f_{\max }}{e \gamma_{i}} .
\end{aligned}
$$

These expressions can be rewritten in dimension form:

$$
\begin{aligned}
& \left|\alpha_{i}\right| \leq \frac{M_{\max }}{2 k_{a}} \\
& \left|\omega_{i}\right| \leq 4 \frac{M_{\max }}{e k_{\omega}} .
\end{aligned}
$$

Hence, expressions that connect attitude and stabilization errors with maximum disturbance torque and control parameters are obtained.

\subsection{Effect of attitude and stabilization errors on trajectory surveying}

As was mentioned above, due to disturbance torques it is impossible to align CF and RF precisely. Moreover, there is a deviation between the real motion of satellite center of mass and the one considered in control loop. All these factors may affect the image obtained by SC: because of attitude and center of mass position errors the satellite surveys not the trajectory but its vicinity, and due to the stabilization and center of mass velocity error obtained image might be blurred.

If only small deviations between CF and RF are considered, transition matrix from IF to $\mathrm{CF}$ can be written as follows: 


$$
\mathbf{K}=\mathbf{F B} \approx\left(\mathbf{E}_{3}+\left(\begin{array}{ccc}
0 & \beta & -\alpha \\
-\beta & 0 & \gamma \\
\alpha & -\gamma & 0
\end{array}\right)\right) \mathbf{B},
$$

where $\alpha, \beta, \gamma$ are Euler angles (rotation sequence 2-3-1). Therefore, expressions that connect basis vectors of $\mathrm{CF}$ and $\mathrm{RF}$ are

$$
\begin{aligned}
& \mathbf{e}_{1}=\mathbf{K}^{T}\left(\begin{array}{lll}
1 & 0 & 0
\end{array}\right)^{T}=\mathbf{e}_{1}^{0}+\beta \mathbf{e}_{2}^{0}-\alpha \mathbf{e}_{3}^{0}=\mathbf{e}_{1}^{0}+\delta \mathbf{e}_{1}, \\
& \mathbf{e}_{2}=\mathbf{K}^{T}\left(\begin{array}{lll}
0 & 1 & 0
\end{array}\right)^{T}=\mathbf{e}_{2}^{0}-\beta \mathbf{e}_{1}^{0}+\gamma \mathbf{e}_{3}^{0}=\mathbf{e}_{2}^{0}+\delta \mathbf{e}_{2}, \\
& \mathbf{e}_{3}=\mathbf{K}^{T}\left(\begin{array}{lll}
0 & 0 & 1
\end{array}\right)^{T}=\mathbf{e}_{3}^{0}+\alpha \mathbf{e}_{1}^{0}-\gamma \mathbf{e}_{2}^{0}=\mathbf{e}_{3}^{0}+\delta \mathbf{e}_{3} .
\end{aligned}
$$

Where $\mathbf{e}_{i}$ are basis vectors of $\mathrm{CF}$, and $\mathbf{e}_{i}^{0}$ are basis vectors of RF.

To determine real observation point $\mathbf{r}_{p}$, i.e. the point satellite "looking" at, and its deviation $\delta \mathbf{r}_{p}$ from the desirable one, it is necessary to find intersection of camera optical axis and Earth surface. To do so, the following quadratic equation must be solved (Earth considered as a spheroid):

$$
\frac{\left(r_{1}+e_{1} l\right)^{2}}{a^{2}}+\frac{\left(r_{2}+e_{2} l\right)^{2}}{a^{2}}+\frac{\left(r_{3}+e_{3} l\right)^{2}}{a^{2}(1-\sigma)^{2}}=1,
$$

where $r_{i}, e_{i}$ are $\mathbf{r}_{s}$ and $\mathbf{e}_{1}$ components in IF, $l$ - unknown value, $a$ - spheroid major semi-axis, $\sigma \approx 1 / 300$ - polar compression. $\mathbf{r}_{p}$ then can be determine as follows:

$$
\mathbf{r}_{p}=\mathbf{r}_{s}+\mathbf{e}_{1} l \text {. }
$$

There are three different cases:

1) Equation (10) has no roots, thus there is no intersection between camera optical axis and Earth surface.

2) Equation has only one root, so the observation point is located on horizon.

3) Equation has two roots. In this case, the smallest root gives us the observation point.

Since $\sigma$ is small, it is possible to omit all terms with $\sigma^{2}$, so (10) can be rewritten as follows:

$$
l^{2}(1+2 \sigma)+2 l\left(\left(\mathbf{r}_{s}, \mathbf{e}_{1}\right)+2 \sigma r_{3} e_{3}\right)+\left|\mathbf{r}_{s}\right|^{2}-a^{2}+2 \sigma r_{3}^{2}=0,
$$

and its roots are 


$$
\begin{aligned}
& l_{1,2}=-\left(\mathbf{r}_{s}, \mathbf{e}_{1}\right) \pm \sqrt{\left(\mathbf{r}_{s}, \mathbf{e}_{1}\right)^{2}-\left|\mathbf{r}_{s}\right|^{2}+a^{2}}+ \\
& +\sigma\left(-2 r_{3} e_{3}+2\left(\mathbf{r}_{s}, \mathbf{e}_{1}\right) \mp \frac{r_{3}^{2}-\left|\mathbf{r}_{s}\right|^{2}+a^{2}+2\left(\mathbf{r}_{s}, \mathbf{e}_{1}\right)^{2}-2 r_{3} e_{3}\left(\mathbf{r}_{s}, \mathbf{e}_{1}\right)}{\sqrt{\left(\mathbf{r}_{s}, \mathbf{e}_{1}\right)^{2}-\left|\mathbf{r}_{s}\right|^{2}+a^{2}}}\right) .
\end{aligned}
$$

One can see, that term with $\sigma$ is two orders smaller than the one without it, so it is reasonable to omit it and consider Earth as a sphere rather than spheroid. In this case first two terms of (11) with consideration of $\mathbf{r}_{s}=\mathbf{r}_{s}^{0}+\delta \mathbf{r}_{s}$ can be rewritten as follows (here only zero and first order of $\delta \mathbf{r}_{s} / r_{s}$ and $\delta \mathbf{e}_{i}$ are considered):

$$
\begin{aligned}
& l=-\left(\mathbf{r}_{s}^{0}+\delta \mathbf{r}_{s}, \mathbf{e}_{1}^{0}+\delta \mathbf{e}_{1}\right)-\sqrt{\left(\mathbf{r}_{s}^{0}+\delta \mathbf{r}_{s}, \mathbf{e}_{1}^{0}+\delta \mathbf{e}_{1}\right)^{2}-\left(\left(\mathbf{r}_{s}^{0}+\delta \mathbf{r}_{s}\right)^{2}-a^{2}\right)}= \\
& =-\left(\mathbf{r}_{s}^{0}, \mathbf{e}_{1}^{0}\right)-\left(\delta \mathbf{r}_{s}, \mathbf{e}_{1}^{0}\right)-\left(\mathbf{r}_{s}^{0}, \delta \mathbf{e}_{1}\right)-\sqrt{\left(\mathbf{r}_{s}^{0}, \mathbf{e}_{1}^{0}\right)^{2}-\left(\left(\mathbf{r}_{s}^{0}\right)^{2}-a^{2}\right)} \times \\
& \times\left(1+2 \frac{\left.\left(\mathbf{r}_{s}^{0}, \mathbf{e}_{1}^{0}\right)\left(\left(\delta \mathbf{r}_{s}, \mathbf{e}_{1}^{0}\right)+\left(\mathbf{r}_{s}^{0}, \delta \mathbf{e}_{1}\right)\right)-\left(\mathbf{r}_{s}^{0}, \delta \mathbf{r}_{s}\right)\right)^{\frac{1}{2}} \approx l^{0}+\delta l}{\left(\mathbf{r}_{s}^{0}, \mathbf{e}_{1}^{0}\right)^{2}-\left(\left(\mathbf{r}_{s}^{0}\right)^{2}-a^{2}\right)}\right. \\
& \delta l=-\left(\delta \mathbf{r}_{s}, \mathbf{e}_{1}^{0}\right)-\left(\mathbf{r}_{s}^{0}, \delta \mathbf{e}_{1}\right)-\frac{\left(\mathbf{r}_{s}^{0}, \mathbf{e}_{1}^{0}\right)\left(\left(\delta \mathbf{r}_{s}, \mathbf{e}_{1}^{0}\right)+\left(\mathbf{r}_{s}^{0}, \delta \mathbf{e}_{1}\right)\right)-\left(\mathbf{r}_{s}^{0}, \delta \mathbf{r}_{s}\right)}{\sqrt{\left(\mathbf{r}_{s}^{0}, \mathbf{e}_{1}^{0}\right)^{2}-\left(\left(\mathbf{r}_{s}^{0}\right)^{2}-a^{2}\right)}} \\
& l^{0}=-\left(\mathbf{r}_{s}^{0}, \mathbf{e}_{1}^{0}\right)-\sqrt{\left(\mathbf{r}_{s}^{0}, \mathbf{e}_{1}^{0}\right)^{2}-\left(\left(\mathbf{r}_{s}^{0}\right)^{2}-a^{2}\right)} .
\end{aligned}
$$

Thus, expression for observation point is

$$
\mathbf{r}_{p}=\mathbf{r}_{s}+\mathbf{e}_{1} l=\mathbf{r}_{s}^{0}+\delta \mathbf{r}_{s}+\mathbf{e}_{1}^{0} l^{0}+\delta \mathbf{e}_{1} l^{0}+\mathbf{e}_{1}^{0} \delta l=\mathbf{r}_{p}^{0}+\delta \mathbf{r}_{s}+\delta \mathbf{e}_{1} l^{0}+\mathbf{e}_{1}^{0} \delta l .
$$

Using notation

$$
\begin{gathered}
\delta \mathbf{x}=\left(\delta \alpha, \delta \beta, \delta \gamma, \delta \omega_{1}, \delta \omega_{2}, \delta \omega_{3}, \delta r_{s}^{1}, \delta r_{s}^{2}, \delta r_{s}^{3}, \delta V_{s}^{1}, \delta V_{s}^{2}, \delta V_{s}^{3}\right)^{T}= \\
=\left(\delta \boldsymbol{\psi}^{T}, \delta \boldsymbol{\omega}^{T}, \delta \mathbf{r}_{s}^{T}, \delta \mathbf{V}_{s}^{T}\right)^{T},
\end{gathered}
$$




$$
\begin{aligned}
& \mathbf{M}=\left[\mathbf{M}_{1-3,1}, \mathbf{M}_{1-3,2}, \mathbf{M}_{1-3,3-6}, \mathbf{M}_{1-3,7-9}, \mathbf{M}_{1-3,10-12}\right] \\
& \mathbf{M}_{1-3,1}=\mathbf{e}_{1}^{0} \frac{\left(\mathbf{r}_{s}^{0}, \mathbf{e}_{1}^{0}\right)\left(\mathbf{r}_{s}^{0}, \mathbf{e}_{3}^{0}\right)}{\sqrt{\left(\mathbf{r}_{s}, \mathbf{e}_{1}^{0}\right)^{2}-\left(\left(\mathbf{r}_{s}^{0}\right)^{2}-R^{2}\right)}}+\mathbf{e}_{1}^{0}\left(\mathbf{r}_{s}, \mathbf{e}_{3}^{0}\right)-\mathbf{e}_{3}^{0} l^{0}, \\
& \mathbf{M}_{1-3,2}=\mathbf{e}_{2}^{0} l^{0}-\mathbf{e}_{1}^{0} \frac{\left(\mathbf{r}_{s}^{0}, \mathbf{e}_{1}^{0}\right)\left(\mathbf{r}_{s}^{0}, \mathbf{e}_{2}^{0}\right)}{\sqrt{\left(\mathbf{r}_{s}^{0}, \mathbf{e}_{1}^{0}\right)^{2}-\left(\left(\mathbf{r}_{s}^{0}\right)^{2}-R^{2}\right)}}-\mathbf{e}_{1}^{0}\left(\mathbf{r}_{s}^{0}, \mathbf{e}_{2}^{0}\right), \\
& \mathbf{M}_{1-3,3-6}=\mathbf{0}_{3 \times 4}, \\
& \mathbf{M}_{1-3,7-9}=\mathbf{E}_{3 \times 3}-\mathbf{e}_{1}^{0}\left(\mathbf{e}_{1}^{0}\right)^{T}+\frac{\mathbf{e}_{1}^{0}\left(\mathbf{r}_{s}^{0}\right)^{T}-\left(\mathbf{r}_{s}^{0}, \mathbf{e}_{1}^{0}\right) \mathbf{e}_{1}^{0}\left(\mathbf{e}_{1}^{0}\right)^{T}}{\sqrt{\left(\mathbf{r}_{s}, \mathbf{e}_{1}^{0}\right)^{2}-\left(\left(\mathbf{r}_{s}^{0}\right)^{2}-R^{2}\right)}} \\
& \mathbf{M}_{1-3,10-12}=\mathbf{0}_{3 \times 3},
\end{aligned}
$$

equation for observation point and its displacement can be written in compact form

$$
\mathbf{r}_{p}=\mathbf{r}_{p}^{0}+\delta \mathbf{r}_{p}, \delta \mathbf{r}_{p}=\mathbf{M} \delta \mathbf{x}
$$

Velocity of observation point relative to satellite is

$$
\mathbf{V}_{r e l}=\mathbf{V}_{p}-\mathbf{V}_{s}-\left(\mathbf{B}^{T} \mathbf{F}^{T} \mathbf{G}^{T} \boldsymbol{\omega}\right) \times\left(\mathbf{r}_{p}-\mathbf{r}_{s}\right)=\boldsymbol{\Omega}_{\mathrm{E}} \times \mathbf{r}_{p}-\mathbf{V}_{s}-\left(\mathbf{B}^{T} \mathbf{F}^{T} \mathbf{G}^{T} \boldsymbol{\omega}\right) \times\left(\mathbf{r}_{p}-\mathbf{r}_{s}\right) .
$$

Matrix $\mathbf{B}^{T} \mathbf{F}^{T} \mathbf{G}^{T}$ is the transition matrix between BF and IF. Considering only small deviations between RF and CF, matrix $\mathbf{F}$ can be written as follows:

$$
\mathbf{F} \approx \mathbf{E}_{3}+\left(\begin{array}{ccc}
0 & \beta & -\alpha \\
-\beta & 0 & \gamma \\
\alpha & -\gamma & 0
\end{array}\right)=\mathbf{E}_{3}+\delta \mathbf{F},
$$

and angular velocity of the satellite is $\boldsymbol{\omega}=\boldsymbol{\omega}^{0}+\delta \boldsymbol{\omega}$. Thus, observation point relative velocity is

$$
\begin{aligned}
\mathbf{V}_{r e l}= & \mathbf{V}_{r e l}^{0}+\delta \mathbf{V}, \mathbf{V}_{r e l}^{0}=\mathbf{\Omega}_{\mathrm{E}} \times \mathbf{r}_{p}^{0}-\mathbf{V}_{s}^{0}-\mathbf{B}^{T} \mathbf{G}^{T} \boldsymbol{\omega}^{0} \times\left(\mathbf{r}_{p}^{0}-\mathbf{r}_{s}^{0}\right), \\
\delta \mathbf{V}= & {\left[\boldsymbol{\Omega}_{E}-\mathbf{B}^{T} \mathbf{G}^{T} \boldsymbol{\omega}^{0}\right]_{\times} \delta \mathbf{r}_{p}-\delta \mathbf{V}_{s}+\left[\mathbf{B}^{T} \mathbf{G}^{T} \boldsymbol{\omega}^{0}\right]_{\times} \delta \mathbf{r}_{s}+} \\
& +\left[\mathbf{r}_{p}^{0}-\mathbf{r}_{s}^{0}\right]_{\times}\left(\mathbf{B}^{T} \delta \mathbf{F}^{T} \mathbf{G}^{T} \boldsymbol{\omega}^{0}+\mathbf{B}^{T} \mathbf{G}^{T} \delta \boldsymbol{\omega}\right) .
\end{aligned}
$$

Expressions for image velocity then can be written as 


$$
\begin{gathered}
\frac{f}{\rho}\left(\mathbf{V}_{r e l}, \mathbf{e}_{3}\right)=\frac{f}{\rho}\left(\left(\delta \mathbf{V}, \mathbf{e}_{3}^{0}\right)+\left(\mathbf{V}_{r e l}^{0}, \delta \mathbf{e}_{3}\right)\right), \\
\frac{f}{\rho}\left(\mathbf{V}_{r e l}, \mathbf{e}_{2}\right)=-\frac{V \rho^{0}}{\rho}+\frac{f}{\rho}\left(\left(\delta \mathbf{V}, \mathbf{e}_{2}^{0}\right)+\left(\mathbf{V}_{r e l}^{0}, \delta \mathbf{e}_{2}\right)\right), \\
\rho^{0}=\left\|\mathbf{r}_{p}^{0}-\mathbf{r}_{s}^{0}\right\|, \frac{1}{\rho}=\frac{1}{\left\|\mathbf{r}_{p}-\mathbf{r}_{s}\right\|} \approx \frac{1}{\rho^{0}}\left(1-\frac{\left(\mathbf{r}_{p}^{0}-\mathbf{r}_{s}^{0}, \delta \mathbf{r}_{p}-\delta \mathbf{r}_{s}\right)}{\left(\rho^{0}\right)^{2}}\right) .
\end{gathered}
$$

Here equations (2) were taken into account:

$$
\begin{gathered}
\left(\mathbf{V}_{r e l}^{0}, \mathbf{e}_{3}^{0}\right)=0, \\
\left(\mathbf{V}_{r e l}^{0}, \mathbf{e}_{2}^{0}\right)=-\frac{V \rho^{0}}{f} .
\end{gathered}
$$

Considering (9) and using notation

$$
\begin{gathered}
\frac{f}{\rho}\left(\mathbf{V}_{r e l}, \mathbf{e}_{i}\right)=V_{i}^{0}+\delta V_{i}, i=2,3, \\
V_{2}^{0}=-V, V_{3}^{0}=0,
\end{gathered}
$$

expressions for deviations in image velocity are represented by

$$
\begin{gathered}
\delta V_{3}=\frac{f}{\rho^{0}}\left(\left(\delta \mathbf{V}, \mathbf{e}_{3}^{0}\right)+\alpha\left(\mathbf{V}_{r e l}^{0}, \mathbf{e}_{1}^{0}\right)+\gamma \frac{V \rho^{0}}{f}\right), \\
\delta V_{2}=\frac{f}{\rho^{0}}\left(\left(\delta \mathbf{V}, \mathbf{e}_{2}^{0}\right)-\beta\left(\mathbf{V}_{r e l}^{0}, \mathbf{e}_{1}^{0}\right)\right)+V \frac{\left(\mathbf{r}_{p}^{0}-\mathbf{r}_{s}^{0}, \delta \mathbf{r}_{p}-\delta \mathbf{r}_{s}\right)}{\left(\rho^{0}\right)^{2}} .
\end{gathered}
$$

Obtained expressions are quite complicated for further analysis. However, if we omit center of mass location and velocity errors (these errors does not depend on attitude control system) and consider the case, when observation point is located not too far from nadir point, i.e.

$$
\frac{r_{s}|\sin \varphi|}{\sqrt{a^{2}-r_{s}^{2} \sin ^{2} \varphi}} \ll 1
$$

where $\varphi$ is the angle between local vertical and radius-vector that connects satellite and observation point, expressions for observation point displacement and image velocity errors can be simplified: 


$$
\begin{gathered}
\left|\delta \mathbf{r}_{p}\right| \approx \rho^{0}|\delta \boldsymbol{\psi}|, \\
\left|\delta V_{3}\right| \approx f|\delta \boldsymbol{\psi}|\left(\left|\boldsymbol{\omega}^{0}\right|+\left|\mathbf{\Omega}_{E}\right|\right)+f|\delta \boldsymbol{\omega}|+V|\delta \boldsymbol{\psi}|, \\
\left|\delta V_{2}\right| \approx f|\delta \boldsymbol{\psi}|\left(\left|\boldsymbol{\omega}^{0}\right|+\left|\mathbf{\Omega}_{E}\right|\right)+f|\delta \boldsymbol{\omega}| .
\end{gathered}
$$

These expressions might be useful on the preliminary design stage to appropriately choose parameters of the attitude determination and control system.

\subsection{Trajectory curvature effect}

Attitude control system can produce only limited torque, which implies constraints on the satellite's maximum angular velocity. If stabilized motion is considered, expression for control torque can be written as follows: (7)

$$
\mathbf{M}_{c t r l}=-\mathbf{M}_{e x t}+\boldsymbol{\omega}_{r e f} \times \mathbf{J} \boldsymbol{\omega}_{r e f}+\mathbf{J} \dot{\boldsymbol{\omega}}_{r e f} .
$$

Consider the case when external torque and derivative of reference angular velocity are much smaller than $\boldsymbol{\omega}_{\text {ref }} \times \mathbf{J} \boldsymbol{\omega}_{\text {ref }}$, one can obtain an estimation for maximum angular velocity that attitude control system could provide:

$$
\left|\omega_{\max }\right| \approx \sqrt{\frac{M_{0}}{\max _{i, j}\left|J_{i i}-J_{j j}\right|}},
$$

where $M_{0}$ is maximum control torque. Constraints on trajectory curvature can be found using expressions for $\mathrm{SC}$ reference angular velocity

$$
\begin{gathered}
\omega_{1}=-\left(\dot{\mathbf{e}}_{3}, \mathbf{e}_{2}\right), \\
\omega_{2}=-\frac{\left(\mathbf{\Omega}_{E} \times \mathbf{N r _ { p }}-\mathbf{V}_{s}, \mathbf{e}_{3}\right)}{\rho}, \\
\omega_{3}=\frac{\left(\boldsymbol{\Omega}_{E} \times \mathbf{N} \mathbf{r}_{p}-\mathbf{V}_{s}, \mathbf{e}_{2}\right)}{\rho}+\frac{V}{f} .
\end{gathered}
$$

First expression can be rewritten in the following form:

$$
\begin{gathered}
\omega_{1}=-\frac{\left(\dot{\mathbf{e}}_{1} \times \mathbf{N} \boldsymbol{\tau}+\mathbf{e}_{1} \times(\dot{\mathbf{N}} \boldsymbol{\tau}+\dot{p} \mathbf{N} \partial \boldsymbol{\tau} / \partial p), \mathbf{e}_{2}\right)}{\left|\mathbf{e}_{1} \times \mathbf{N} \boldsymbol{\tau}\right|}= \\
=\omega_{2} \frac{\left(\mathbf{e}_{1}, \mathbf{N} \boldsymbol{\tau}\right)}{\left|\mathbf{e}_{1} \times \mathbf{N} \boldsymbol{\tau}\right|}-\frac{\left(\boldsymbol{\Omega}_{E} \times \mathbf{N} \boldsymbol{\tau}, \mathbf{e}_{3}\right)}{\left|\mathbf{e}_{1} \times \mathbf{N} \boldsymbol{\tau}\right|}-\dot{p} \frac{\left(\mathbf{N} \partial \boldsymbol{\tau} / \partial p, \mathbf{e}_{1} \times \mathbf{N} \boldsymbol{\tau}\right)}{\left|\mathbf{e}_{1} \times \mathbf{N} \boldsymbol{\tau}\right|^{2}} .
\end{gathered}
$$

Introducing new variable $\zeta$ - angle between $\mathbf{e}_{1}$ and $\mathbf{N} \boldsymbol{\tau},-$ and taking into account expressions for trajectory curvature 


$$
\boldsymbol{\kappa}=\frac{\mathbf{N} \boldsymbol{\tau} \times \mathbf{N} \partial \boldsymbol{\tau} / \partial p}{|\mathbf{N} \boldsymbol{\tau}|^{3}}
$$

equation for $\omega_{1}$ can be represented by

$$
\omega_{1}=\omega_{2} \operatorname{ctg}(\zeta)-\left(\boldsymbol{\Omega}_{E}, \mathbf{e}_{1}\right)+\left(\boldsymbol{\Omega}_{E}, \mathbf{e}_{2}\right) \operatorname{ctg}(\zeta)-\rho \frac{V}{f} \frac{\left(\mathbf{e}_{1}, \mathbf{\kappa}\right)}{\sin ^{3} \zeta} .
$$

Small value of $\sin \zeta$ corresponds to surveying near horizon, that is practically useless due to the significant geometric distortion. Therefore, it is reasonable to bound minimum value of $\sin \zeta$. For example, if $\sin \zeta \geq \sqrt{3} / 2$, one can get an upper bound for trajectory curvature:

$$
\kappa \leq f 3 \sqrt{3} \frac{\left(\omega_{\max }-2 \Omega_{E}\right)}{8 \rho V} .
$$

If this inequality is satisfied, it is possible to shoot trajectory.

\section{Simulation}

To verify obtained estimations and algorithms the following mission was simulated (chosen parameters are based on TabletSat-Aurora satellite developed by SPUTNIX, which was launched on 19 June 2014):

1. SC tensor of inertia $\mathbf{J}=\left(\begin{array}{ccc}0.7 & 0.002 & 0.005 \\ 0.002 & 0.579 & 0.009 \\ 0.005 & 0.009 & 0.5\end{array}\right)$.

2. Image velocity $V=50 \mathrm{~mm} / \mathrm{s}$

3. Camera focal length is $6 \mathrm{~m}$.

4. SC moves on circular orbit with $7000 \mathrm{~km}$ radius.

5. Maximum control torque is $10^{-3} \mathrm{~N} \cdot \mathrm{m}$, and minimum is $10^{-7} \mathrm{~N} \cdot \mathrm{m}$.

Curvature radius in accordance with (12) is

$$
r_{\text {curv }} \geq 1000 \mathrm{~km} \text {. }
$$

In this simulation Earth was considered as Krasovsky spheroid, so every point can be determined by its latitude $\varphi$ and longitude $\lambda$ :

$$
\begin{gathered}
\mathbf{r}_{p}(p)=h(\varphi)\left(\begin{array}{c}
\cos \lambda \cos \varphi \\
\sin \lambda \cos \varphi \\
\sin \varphi
\end{array}\right), \\
h(\varphi)=\frac{1}{\sqrt{\frac{\cos ^{2} \varphi}{a^{2}}+\frac{\sin ^{2} \varphi}{b^{2}}}} .
\end{gathered}
$$


Transition matrix $\mathbf{N}$ from GF to IF is

$$
\mathbf{N}=\left(\begin{array}{ccc}
\cos \left(\lambda_{0}+\Omega_{E} t\right) & -\sin \left(\lambda_{0}+\Omega_{E} t\right) & 0 \\
\sin \left(\lambda_{0}+\Omega_{E} t\right) & \cos \left(\lambda_{0}+\Omega_{E} t\right) & 0 \\
0 & 0 & 1
\end{array}\right)
$$

Thus, if longitude and latitude are three times continuously differentiable functions, $\mathbf{r}_{p}(p)$ also will be three times continuously differentiable. In general splines, Bezier curves or B-splines[12] can be used to construct functions with necessary function, but in this simulation $\varphi$ and $\lambda$ are described as follows:

$$
\varphi=\frac{\pi}{2}-k p, \lambda=\alpha \frac{\sin (l p)}{\sin (k p)},
$$

where $\alpha, l, k$ are some constants, $p$ - trajectory parameters. Trajectory in case when $\alpha=0.05, l=1000, k=100$ is represented in Fig. 3 .

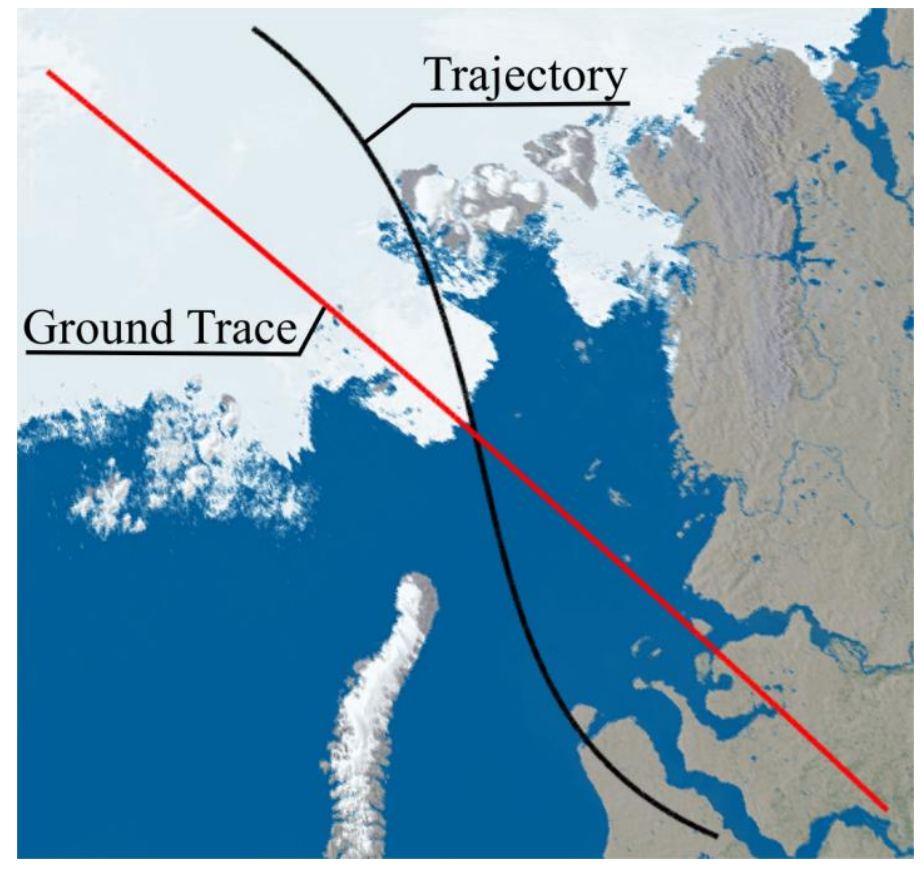

Fig. 3. Trajectory and ground trace

$\mathrm{BF}$ and $\mathrm{CF}$ are considered the same, i.e $\mathbf{G}=\mathbf{E}_{3}$. Also, at start time BF aligned with RF and relative angular velocity is equal to zero. Gravitational and aerodynamic torques are considered as disturbances. Simulations results are presented in Fig. 4-10. 


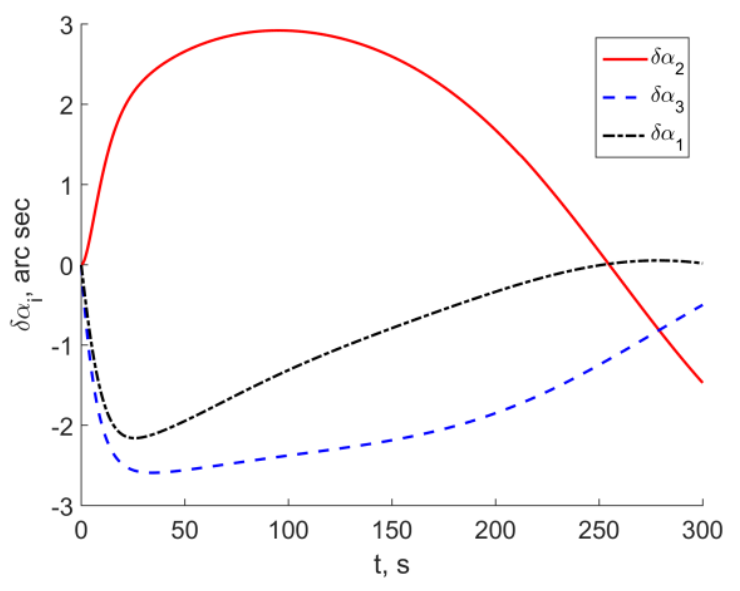

Fig. 4. Attitude error, Euler angles

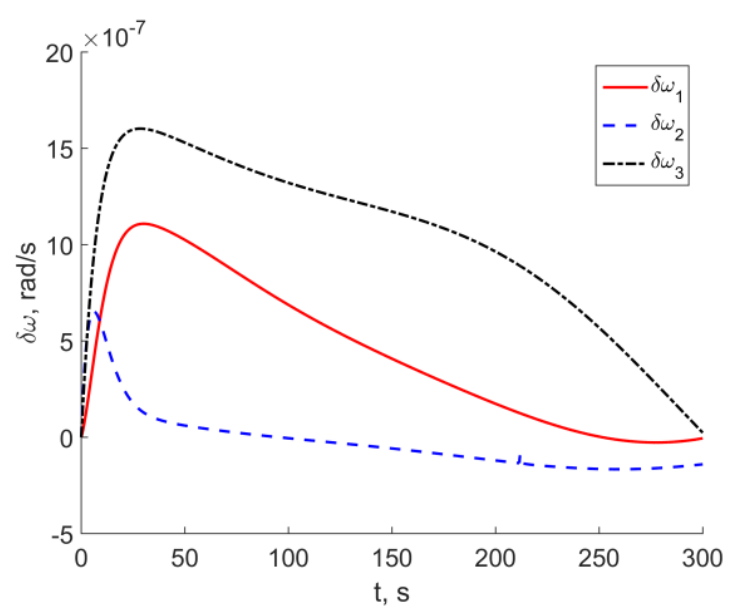

Fig. 6. Relative angular velocity

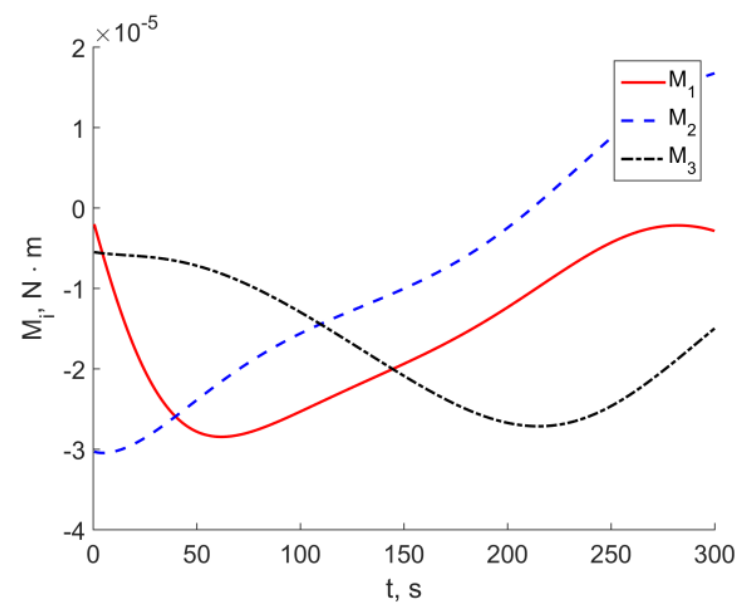

Fig. 8. Control torque

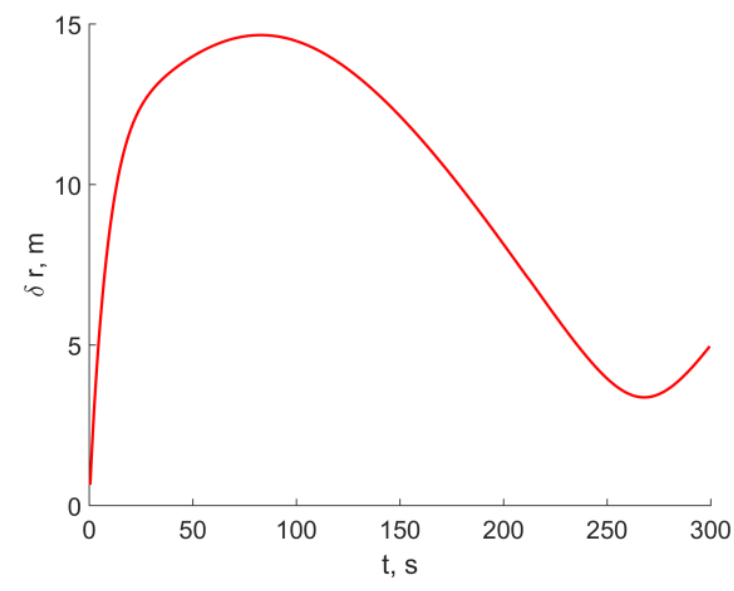

Fig. 5. Observation point displacement

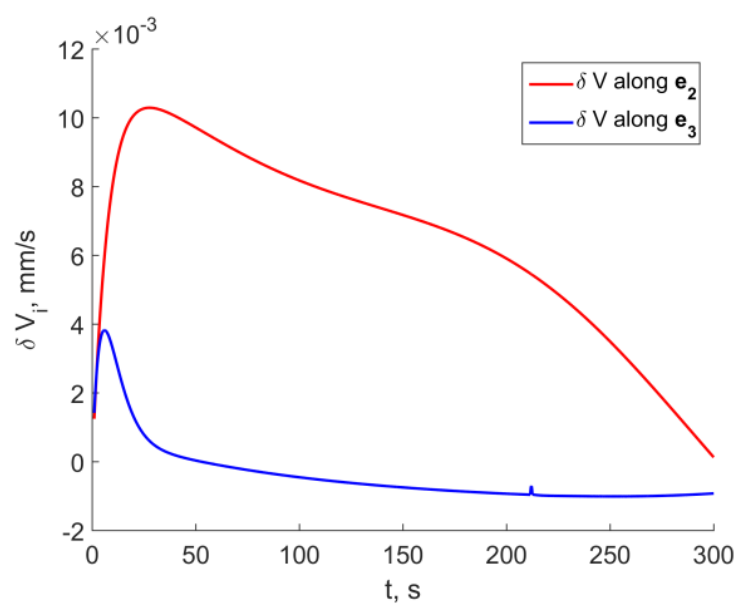

Fig. 7. Image velocity error

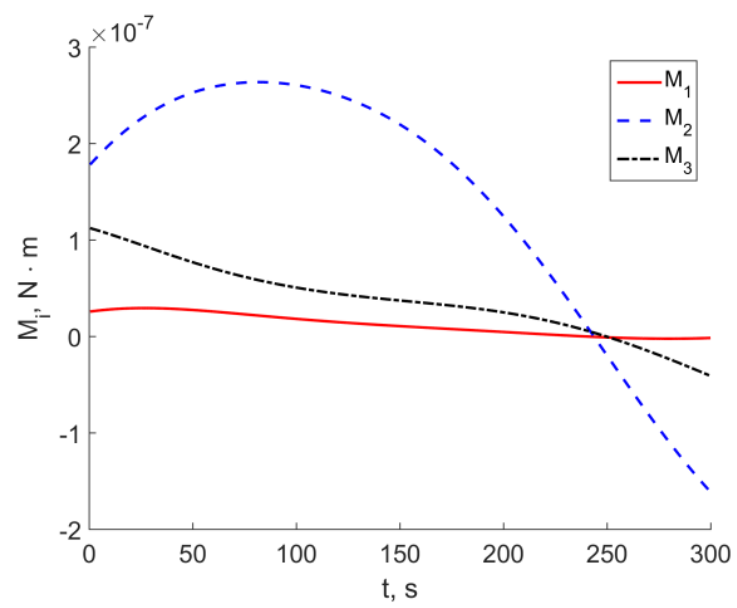

Fig. 9. Disturbance torque

As shown on Fig. 7, even in the presence of disturbances, image velocity error is small. Observation point displacement is less than $15 \mathrm{~m}$, which is agreed with 
accuracy of Russian remote sensing satellite Resurs-P. Small picks on Fig. 6 and 7 occurred because the necessary control torque is too small to produce it.

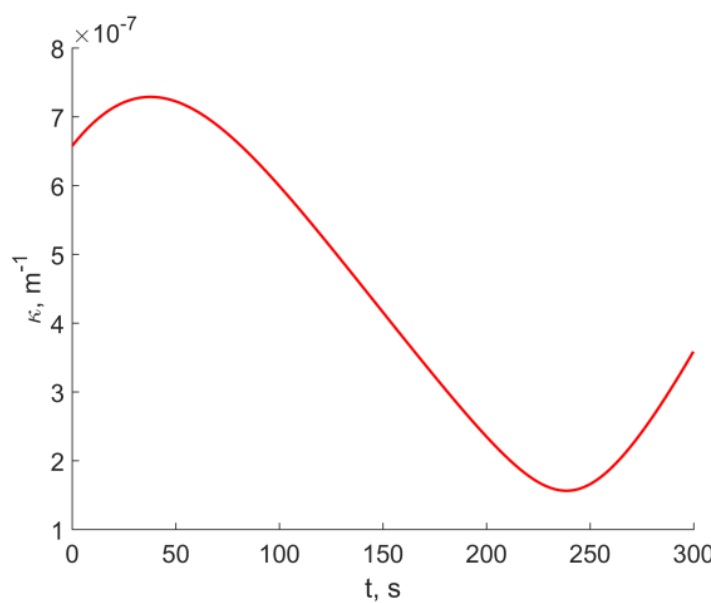

Fig. 10. Trajectory curvature

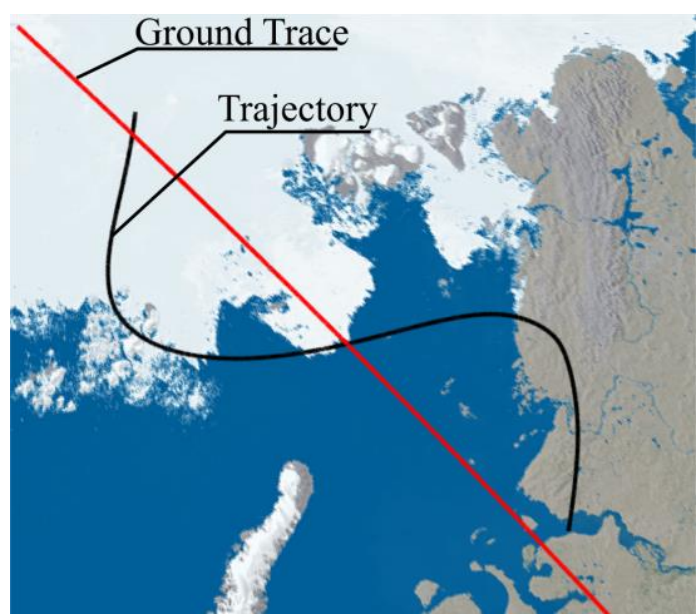

Fig. 11. Trajectory with greater curvature

As shown on Fig. 10, trajectory curvature is less than $10^{-6}$, which is satisfy an estimation obtained earlier. Consider trajectory with greater curvature. To do so, let $l$ be equal to 3000 in (13). Simulation results are presented in Fig. 12-16

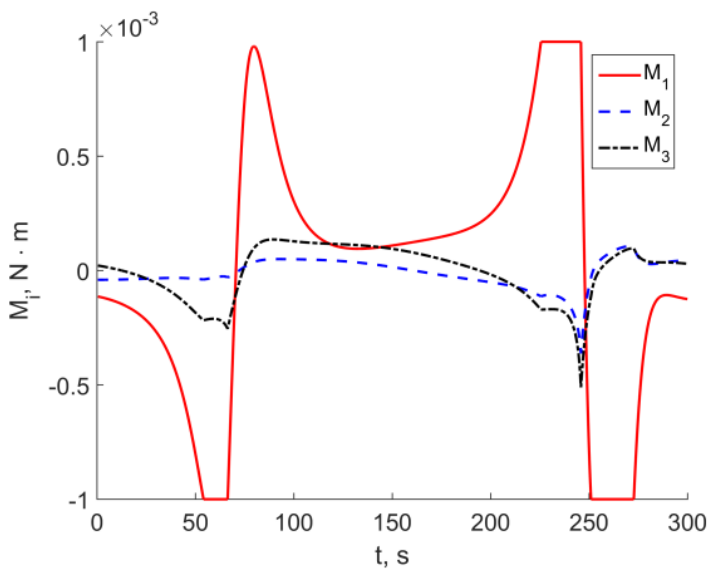

Fig. 12. Control torque

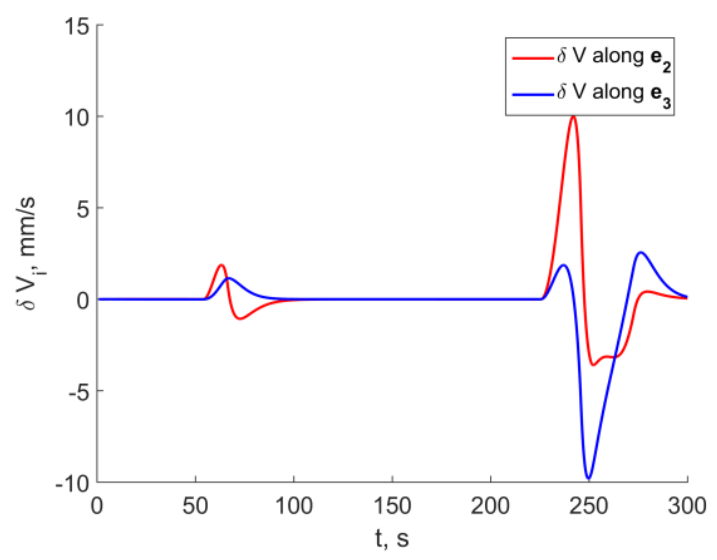

Fig. 14. Image velocity error

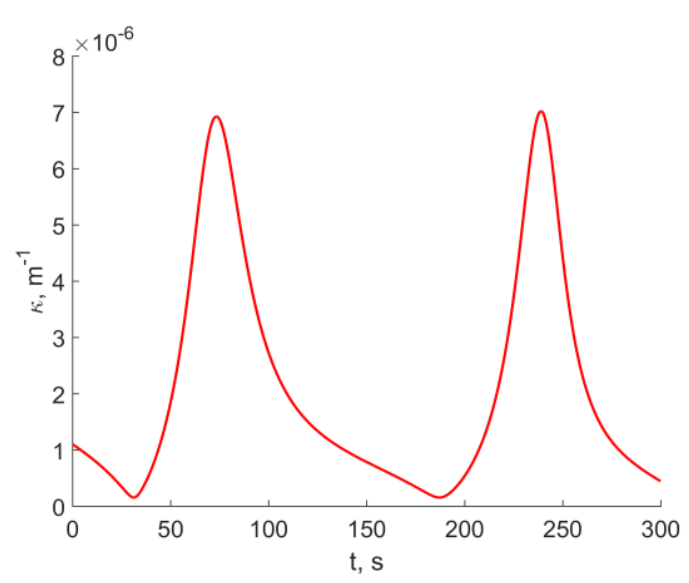

Fig. 13. Trajectory curvature

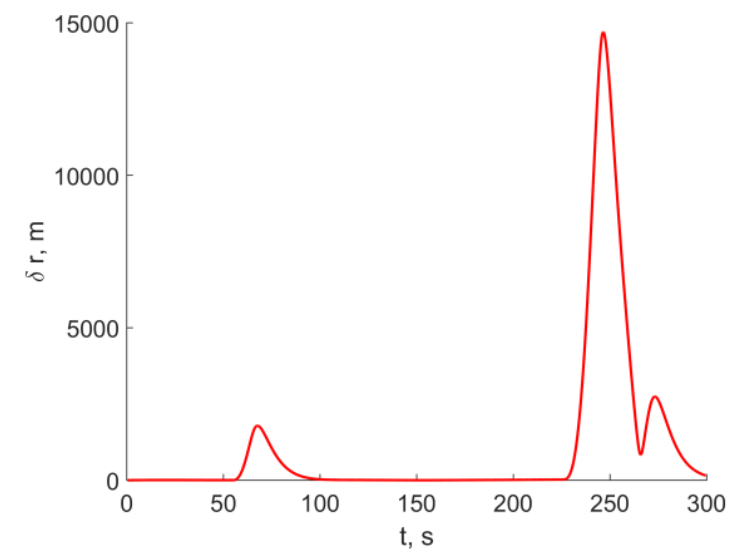

Fig. 15. Observation point displacement 
From these figures one can see that curvature may greatly affect observation point displacement and image velocity error, as control system can not provide the necessary control torque, as shown on Fig. 12.

\section{Conclusion}

An algorithm of angular motion synthesis for remote sensing satellite is suggested. This algorithm provides shooting of complex routes on the Erath surface. Problem of realization of such angular motion in the presence of disturbances is considered. Effect of these disturbances on attitude and stabilization errors is studied. Expressions that link these errors with observation point displacement and image velocity errors are obtained. Trajectory curvature limitations are suggested.

This work is supported by RFBR grants 16-01-00739, 15-31-20058

Authors thank M.Yu. Ovchinnikov for his attention to this work and very useful comments. 


\section{References}

1. Resurs-P

[Electronic

resource].

URL:

http://en.samspace.ru/products/earth_remote_sensing_satellites/ka_resurs_p/ (accessed: 08.04.2016).

2. WorldView

[Electronic

resource].

URL:

http://www.satimagingcorp.com/satellite-sensors/worldview-3/

(accessed: 08.04.2016).

3. Lelong P., Lemercier C., Cheganças J. AstroBus S, the high performance and competetive Small Satellites platform for Earth Observation // 10th IAA Symposium on Small Satellites for Earth Observation / ed. Sandau R., Roser H.-P., Valenzuela A. 2015. P. 15-18.

4. Somov S.E., Butyrin S.A., Somova T.E. Remote sensing satellite targeting law optimization, visualisation and animation of its motion // Proc. XII All-Russian Conf. Control Probl. 2014. P. 3489-3500.

5. Butyrin S.A. Kinematic synthesis of the spacecraft programmed attitude motion at the Earth optic-electronic survey // Proc. Samara Sci. Cent. Russ. Acad. Sci. 2007. Vol. 9, № 3. P. 664-670.

6. Butyrin S.A. Program complex for optic-electronic survey routes computation and visualization // Proc. Samara State Tech. Univ. 2007. Vol. 2. P. 11-17.

7. Wie B., Lu J. Feedback control logic for spacecraft eigenaxis rotations under slew rate and control constraints // J. Guid. Control. Dyn. 1995. Vol. 18, № 6. P. 1372-1379.

8. Wie B., Barba P.M. Quaternion feedback for spacecraft large angle maneuvers. // J. Guid. Control. Dyn. 1985. Vol. 8, № 3. P. 360-365.

9. Tsiotras P. New Control Laws for the Attitude Stabilization of Rigid Bodies // 13th IFAC Symposium on Automatic Control in Aerospace. 1994. P. 316-321.

10. Barbashin E.A. Introduction to the theory of stability. Wolters-Noordhoff, 1970.

11. Malkin I.G. Some problems in the theory of nonlinear oscillations. United States Atomic Energy Commission, Technical Information Service, 1959.

12. Rogers D., Adams A. Mathematical elements for computer graphics. McGrawHill Higher Education, 1989. 


\section{Table of contents}

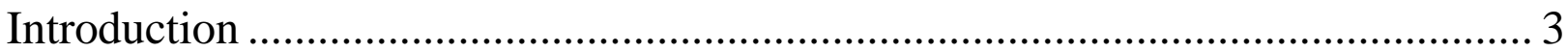

1. Problem statement and coordinate systems ……......................................... 3

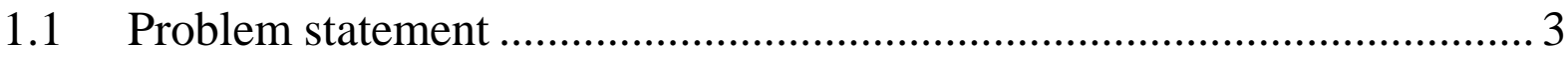

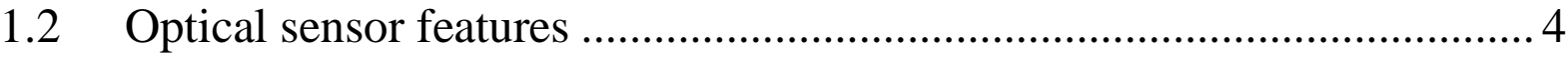

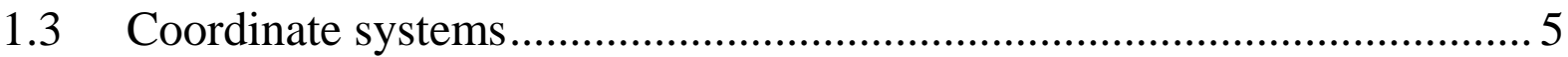

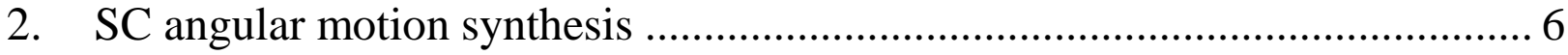

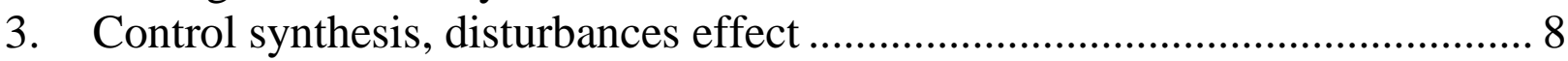

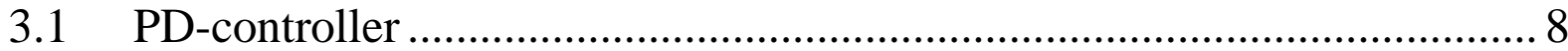

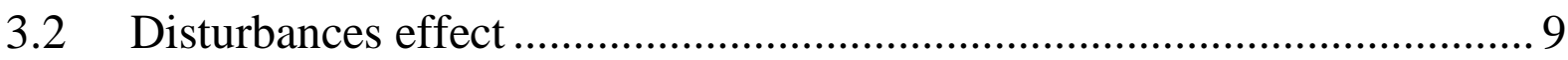

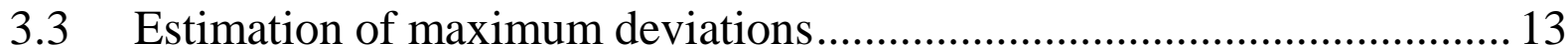

3.4 Effect of attitude and stabilization errors on trajectory shooting ............. 16

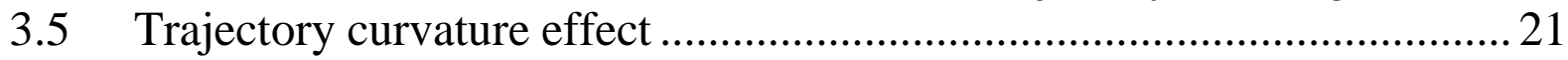

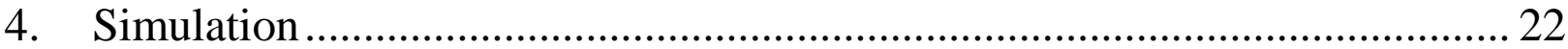

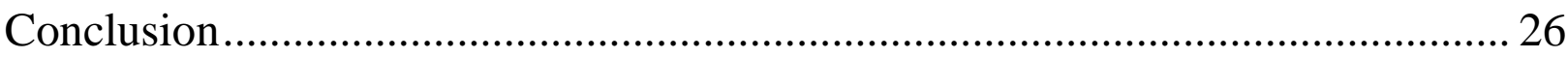

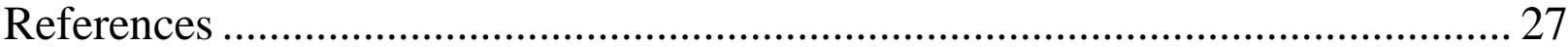

